\title{
NUCLEAR ENGINEERING ENROLLMENTS AND DEGREES, 1994
}

\section{APPENDIXES}

Prepared by:

Oak Ridge Institute for Science and Education

Science/Engineering Education Division

Analysis and Evaluation Programs

Labor and Policy Studies Group

Oak Ridge, Tennessee 37831-0117

Prepared for:

U.S. Department of Energy

Office of Science Education Programs

Washington, D.C. 20585

and

U.S. Nuclear Regulatory Commission

Office of Personnel

Washington, D.C. 20426

May 1995

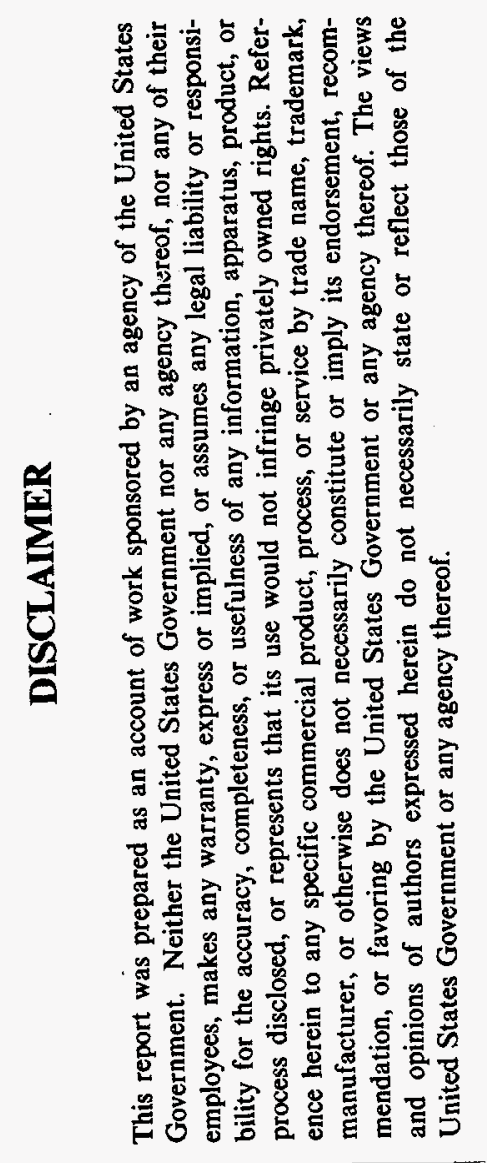

This report is based on work performed for the U.S. Department of Energy and the U.S. Nuclear Regulatory Commission under Contract NO. DE-AC05-760R00033. 


\section{DISCLAIMER}

Portions of this document may be illegible in electronic image products. Images are produced from the best available original document. 


\section{TABLE OF CONTENTS}

\section{APPENDIXES}

Appendix A. Statistical Tables

A-1 Nuclear Engineering Degrees Granted by Institution, 1984-1994 . . . . . . . . . . . . . 3

A-2 Total Enrollment of Full-Time and Part-Time Students in Nuclear Engineering, Fall 1984 through Fall $1994 \ldots \ldots \ldots \ldots \ldots \ldots \ldots \ldots \ldots \ldots$. . . . . . . . . . . . . . . . . . . .

A-3 Nuclear Engineering Enrollments and Degrees in Subfields by State and Institution,

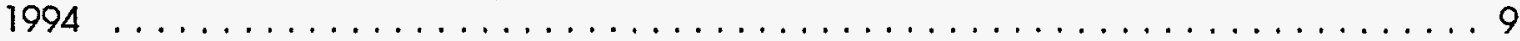

A-4 Nuclear Engineering Enrollments and Degrees by Subfield, $1994 \ldots \ldots \ldots \ldots$

A-5 Undergraduate, Master's, and Doctoral Enrollments and Degrees in Nuclear Engineering, Percent Distribution, $1990-1994 \ldots \ldots \ldots \ldots \ldots \ldots \ldots \ldots \ldots$. . . . . . . . . . . . . .

A-6 Foreign National, Female, and Minority Participation in Nuclear Engineering Programs, 1988-1994

A-7 Nuclear Engineering: Foreign National, Female, and Minority Enrollments and

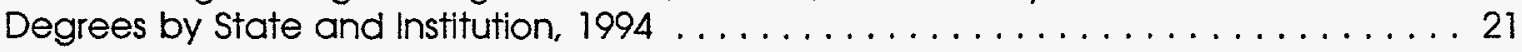

A-8 Total Nuclear Engineering Enrollments and Degrees by Ethnicity and Gender, 1994 .. 34

A-9 Employment or Postgraduation Plans of Nuclear Engineering Graduates by

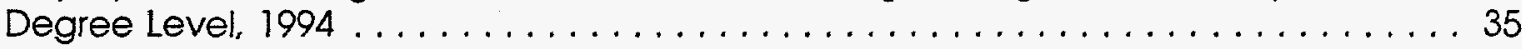

A-10 Nuclear Engineering Enrollments and Degrees by Survey Region and State, 1994 . . 36

A-11 Nuclear Engineering Degrees as a Percentage of All Engineering Degrees by Degree Level, 1974-1994

A-12 Institutions with Undergraduate and Graduate Programs or Options in Nuclear

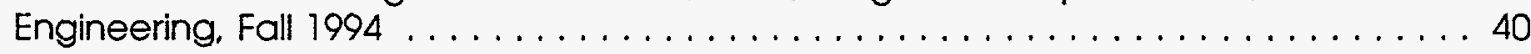

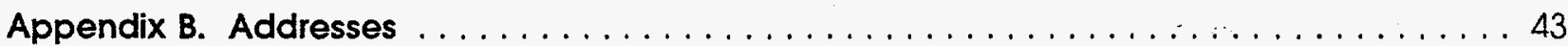

B-1 Addresses of Institutions in Survey Universe, $1994 \ldots \ldots \ldots \ldots \ldots \ldots \ldots$

Appendix C. Survey Materials $\ldots \ldots \ldots \ldots \ldots \ldots \ldots \ldots \ldots \ldots \ldots \ldots \ldots \ldots$ 
APPENDIX A. STATISTICAL TABLES 
APPENDIX A-1

NUCLEAR ENGINEERING

DEGREES GRANTED BY INSTITUTION, 1984-1994

$B=$ Bachelor's

$M=$ Master's

$\mathrm{D}=$ Doctoral

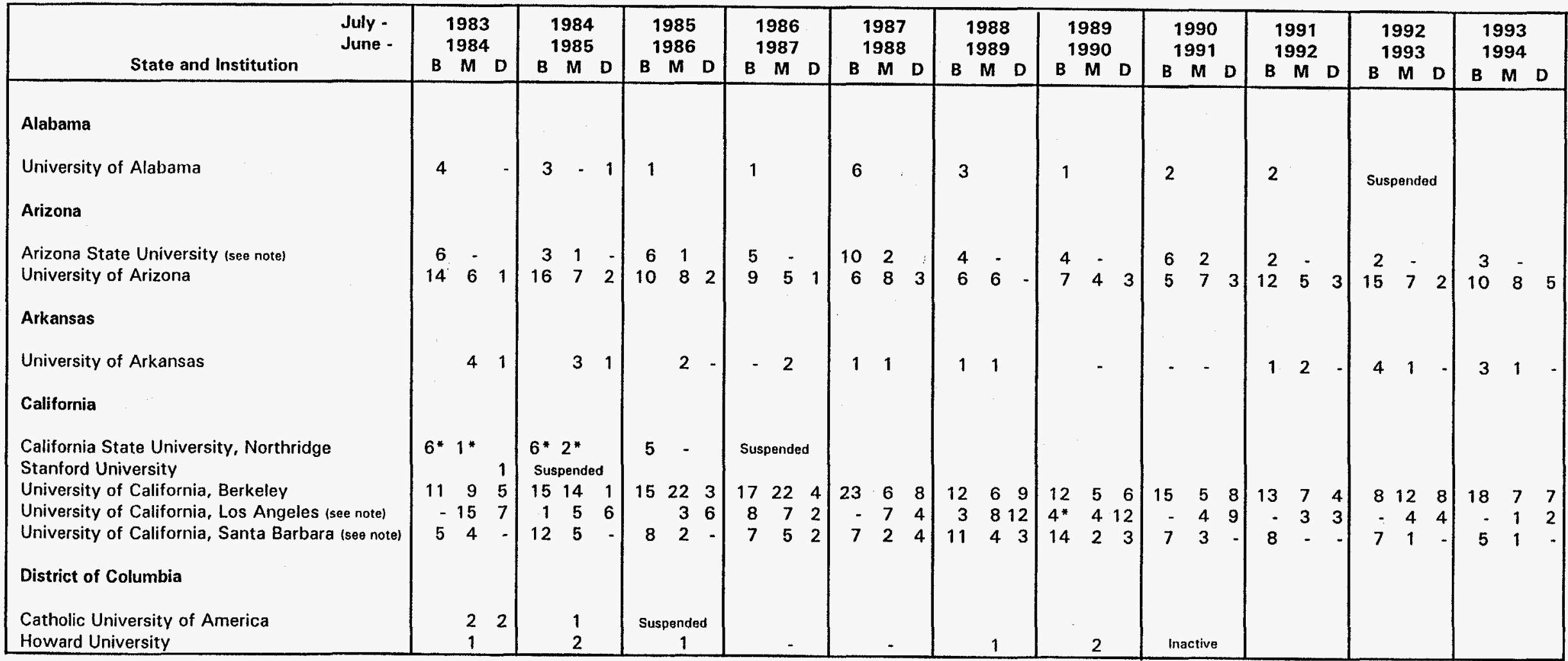

*Estimated.

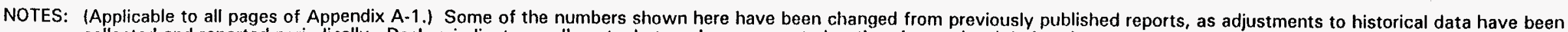
collected and reported periodically. Dashes indicate enrollments, but no degrees granted at that degeee level during the year.

Arizona State University's program has been suspended. Students are being allowed to complete their degrees.

University of California at Los Angeles is unable to report BS degrees, or estimate with any accuracy, at the present time.

The nuclear emphasis at the University of California, Santa Barbara has been suspended. Students are being allowed to complete their degrees. 
APPENDIX A-1 (Continued)

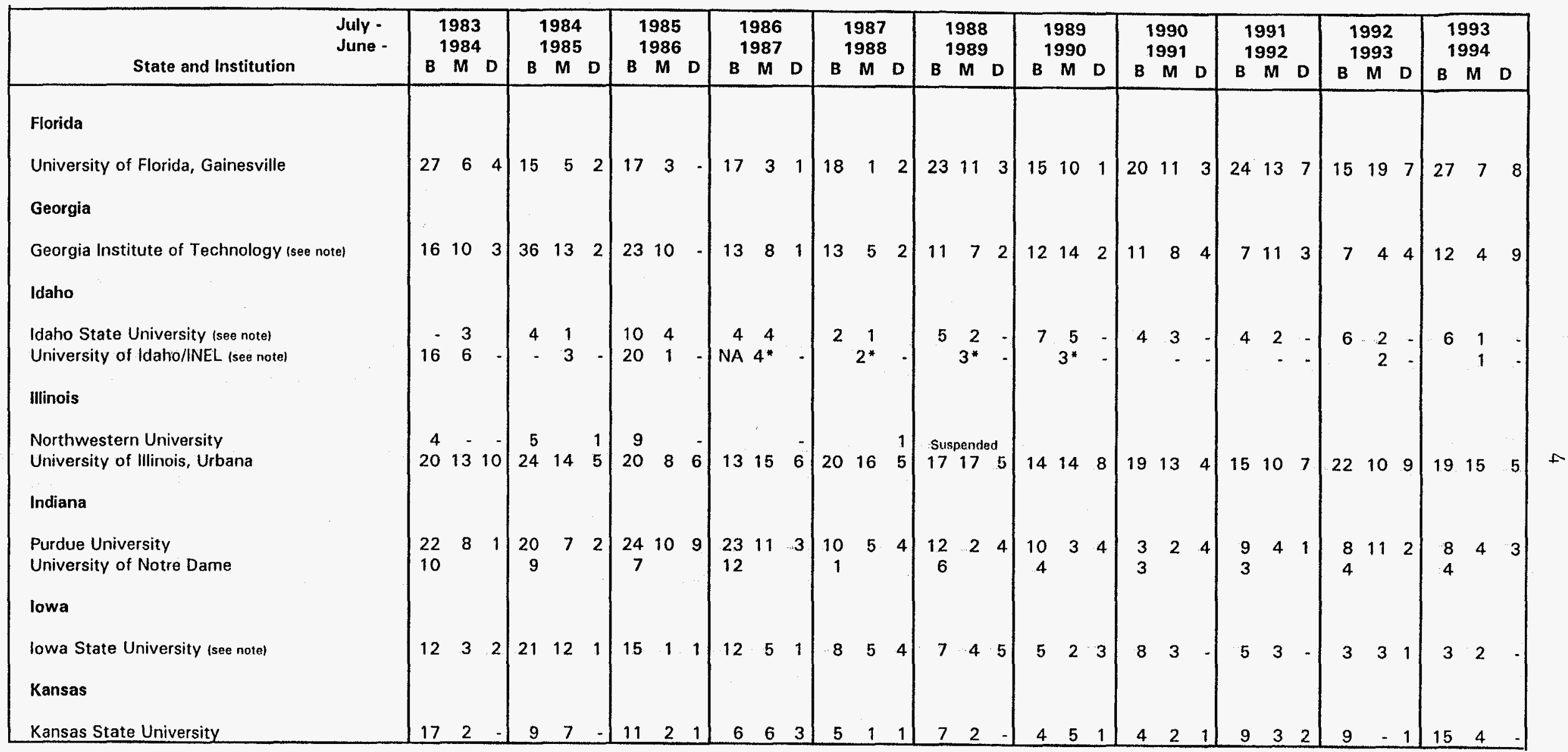

*Estimated.

NOTES: Georgia Institute of Technology has recently relcassified their Ph.D. health physics degrees to nuclear engineering degrees.

The data for the Idaho State University and the University of Idaho include students in the Joint Nuclear Engineering Program which is a cooperative venture with the Idaho National Engineering Laboratory that began in 1989.

lowa State University's undergraduate program has been suspended. Last degrees granted in 1994. 
APPENDIX A-1 (Continued)

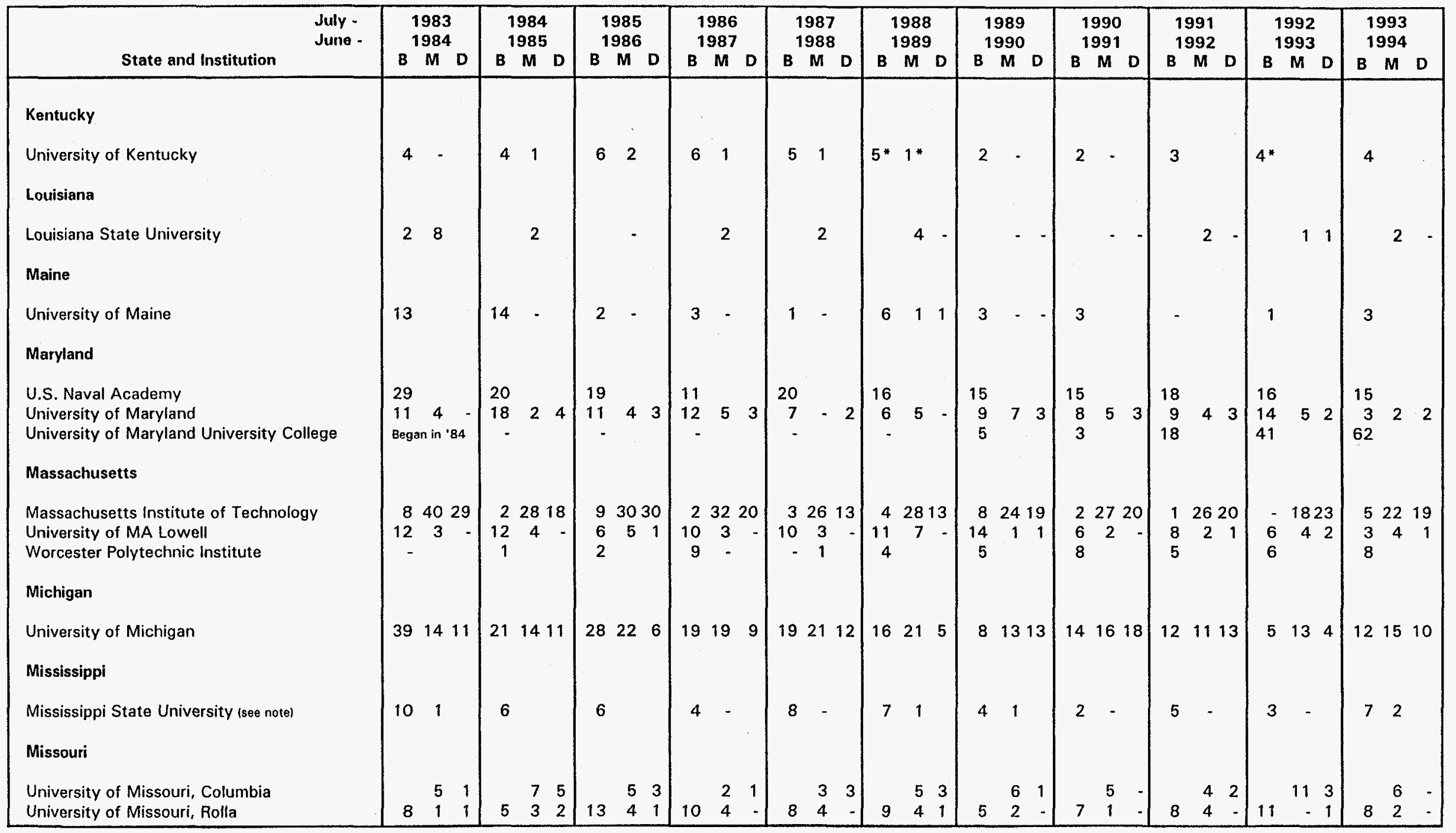

*Estimated.

NOTE: Mississippi State University's program has been suspended. Students are being allowed to complete their degrees. 
6

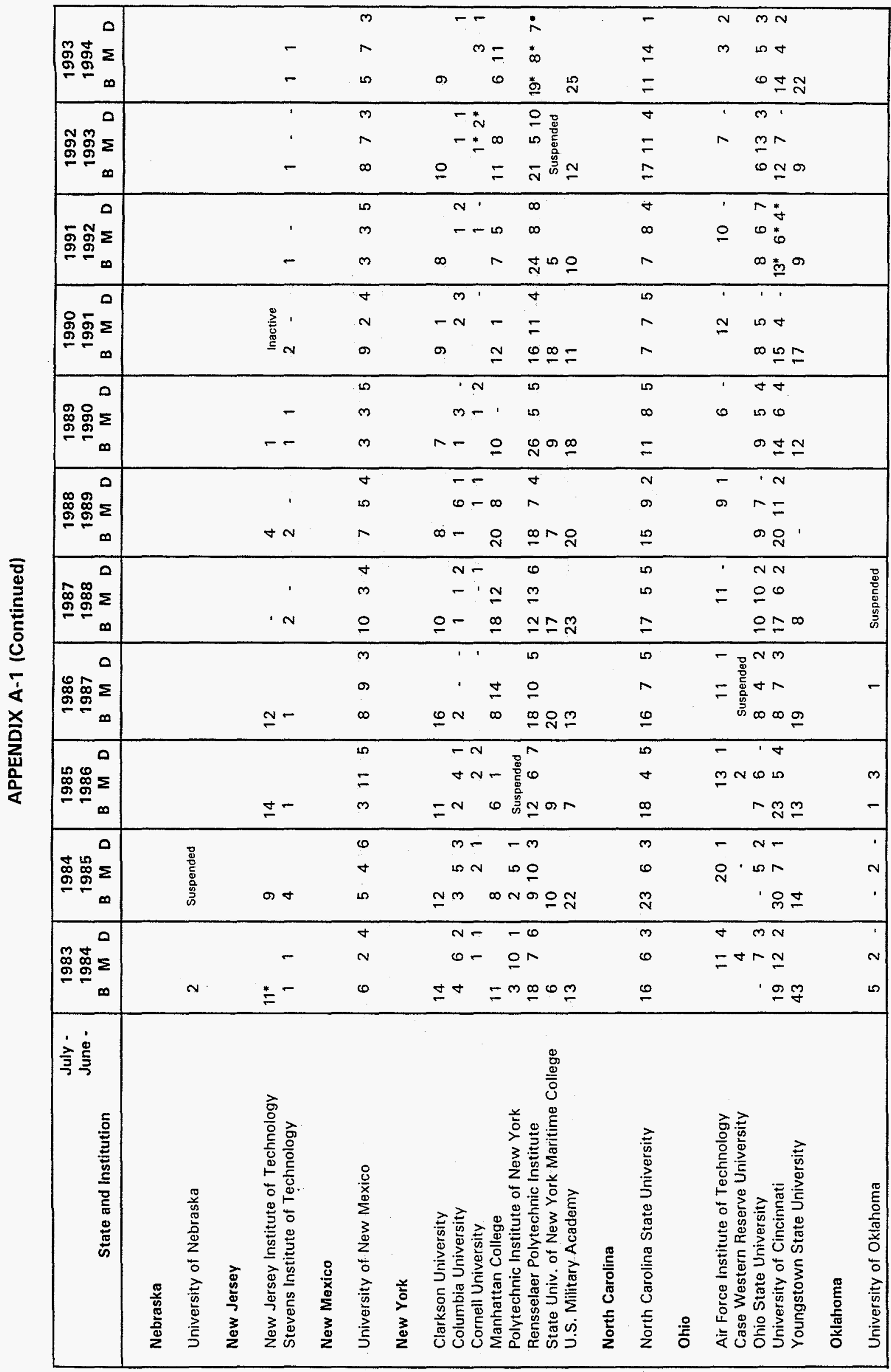




\section{APPENDIX A-1 (Continued)}

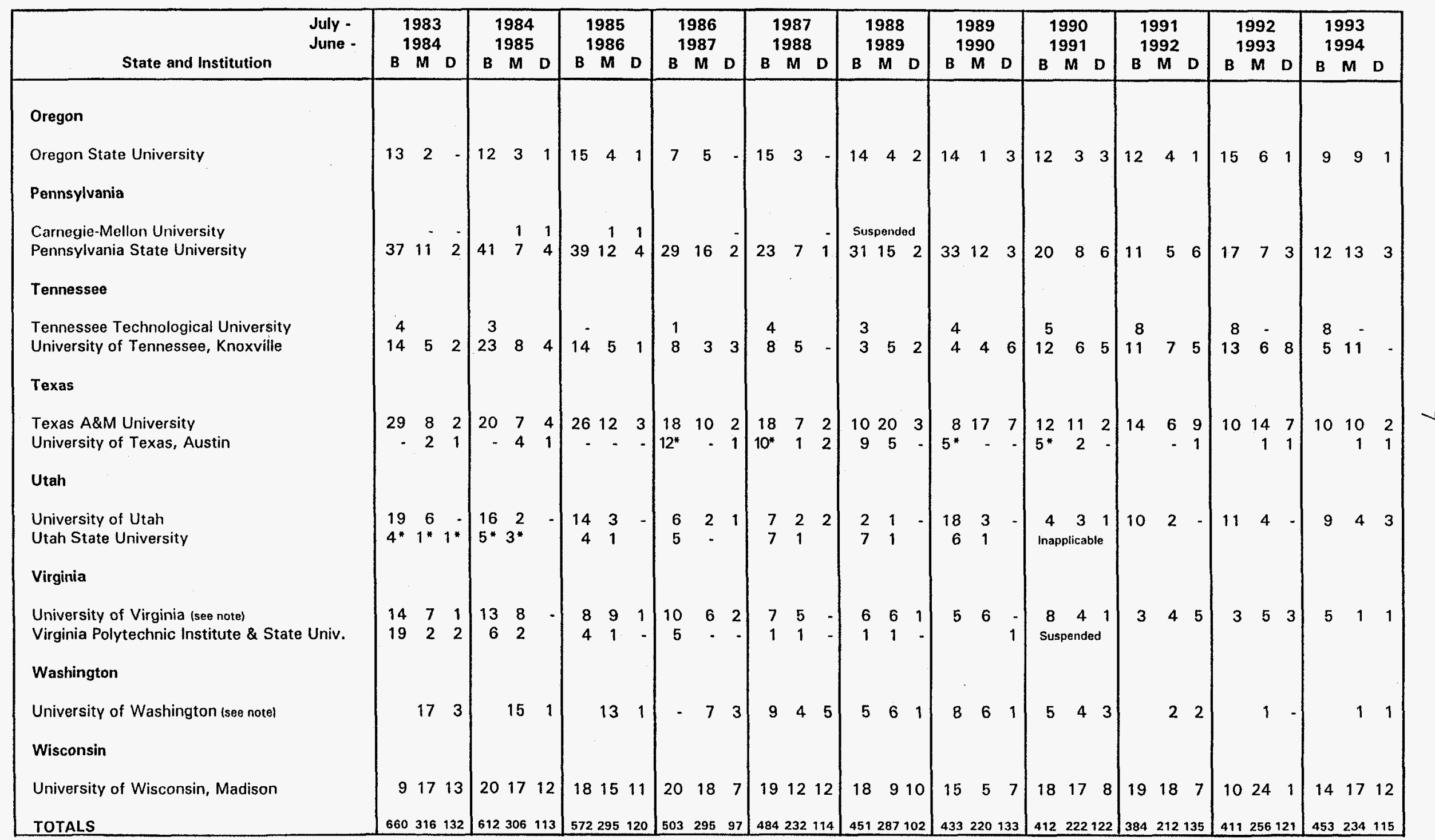

*Estimated.

NOTES: The University of Virginia's undergraduate program has been suspended. Students are being allowed to complete their degrees.

The University of Washington's program has been suspended. Students are being allowed to complete their degrees

Source: U.S. Department of Energy. 


\section{FT $=$ FULL-TIME
PI $=$ PART-TIME}

\begin{tabular}{|c|c|c|c|c|c|c|c|c|c|c|c|c|c|c|c|}
\hline \multirow[b]{3}{*}{ YEAR } & \multicolumn{6}{|c|}{ UNDERGRADUATE } & \multicolumn{6}{|c|}{ GRADUATE } & \multirow{2}{*}{\multicolumn{3}{|c|}{$\begin{array}{c}\text { TOTAL } \\
\text { ENROLLMENT }\end{array}$}} \\
\hline & \multicolumn{3}{|c|}{ JUNIORS } & \multicolumn{3}{|c|}{ SENIORS } & \multicolumn{3}{|c|}{ MASTER'S } & \multicolumn{3}{|c|}{ DOCIORAL } & & & \\
\hline & $F$ & PT & TOTAL & $F T$ & PT & TOTAL & $F$ & PT & TOTAL & $F T$ & PI & TOTAL & FT & PI & TOTAL \\
\hline 1984 & 708 & 23 & 731 & 917 & 15 & 932 & 691 & 233 & 924 & 483 & 76 & 559 & 2799 & 347 & 3146 \\
\hline 1985 & 680 & 25 & 705 & 804 & 16 & 820 & 601 & 234 & 835 & 499 & 83 & 582 & 2584 & 358 & 2942 \\
\hline 1986 & 635 & 80 & 715 & 687 & 27 & 714 & 626 & 180 & 806 & 471 & 78 & 549 & 2419 & 365 & 2784 \\
\hline 1987 & 602 & 91 & 693 & 679 & 21 & 700 & 611 & 181 & 792 & 529 & 84 & 613 & 2421 & 377 & 2798 \\
\hline 1988 & 537 & 164 & 701 & 637 & 51 & 688 & 574 & 176 & 750 & 577 & 70 & 647 & 2325 & 461 & 2786 \\
\hline 1989 & 533 & 182 & 715 & 643 & 56 & 699 & 606 & 151 & 757 & 601 & 69 & 670 & 2383 & 458 & 2841 \\
\hline 1990 & 518 & 181 & 699 & 629 & 70 & 699 & 544 & 178 & 722 & 620 & 81 & 701 & 2311 & 510 & 2821 \\
\hline 1991 & 445 & 242 & 687 & 556 & 139 & 695 & 559 & 225 & 784 & 593 & 106 & 699 & 2153 & 712 & 2865 \\
\hline 1992 & 488 & 282 & 770 & 608 & 119 & 727 & 584 & 231 & 815 & 570 & 76 & 646 & 2250 & 708 & 2958 \\
\hline 1993 & 477 & 213 & 690 & 580 & 115 & 695 & 559 & 216 & 775 & 591 & 97 & 688 & 2207 & 641 & 2848 \\
\hline 1994 & 346 & 178 & 524 & 579 & 135 & 714 & 509 & 217 & 726 & 536 & 102 & 638 & 1970 & 632 & 2602 \\
\hline
\end{tabular}

surce: U.S. Department of Energy 
APPENDIX A-3

NUCLEAR ENGINEERING ENROLLMENTS AND DEGREES

IN SUBFIELDS BY STATE AND INSTITUTION, 1994

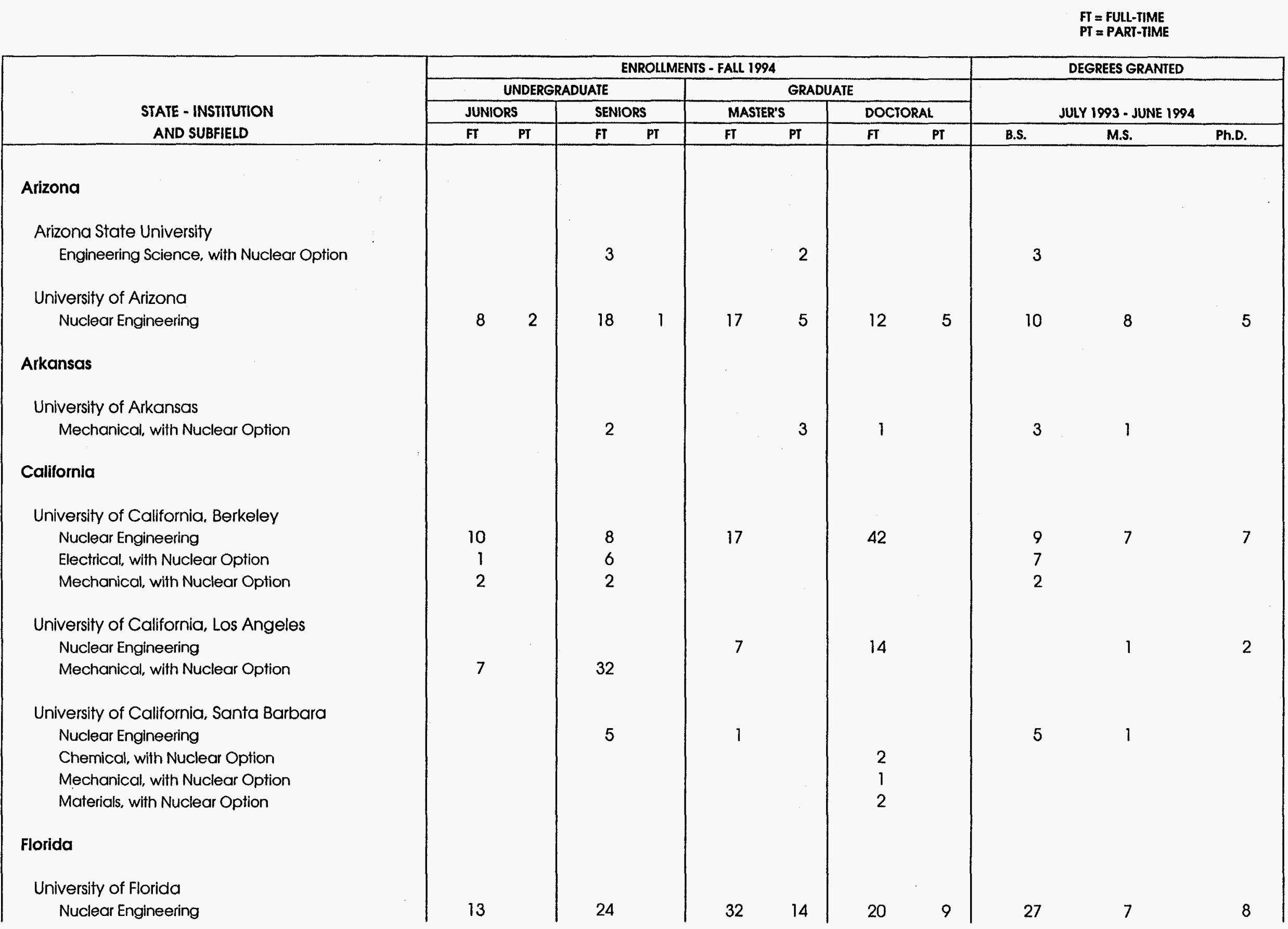


APPENDIX A-3

NUCLEAR ENGINEERING ENROLIMENTS AND DEGREES

IN SUBFIELDS BY STATE AND INSTITUTION, 1994

FT = FULL-TIME

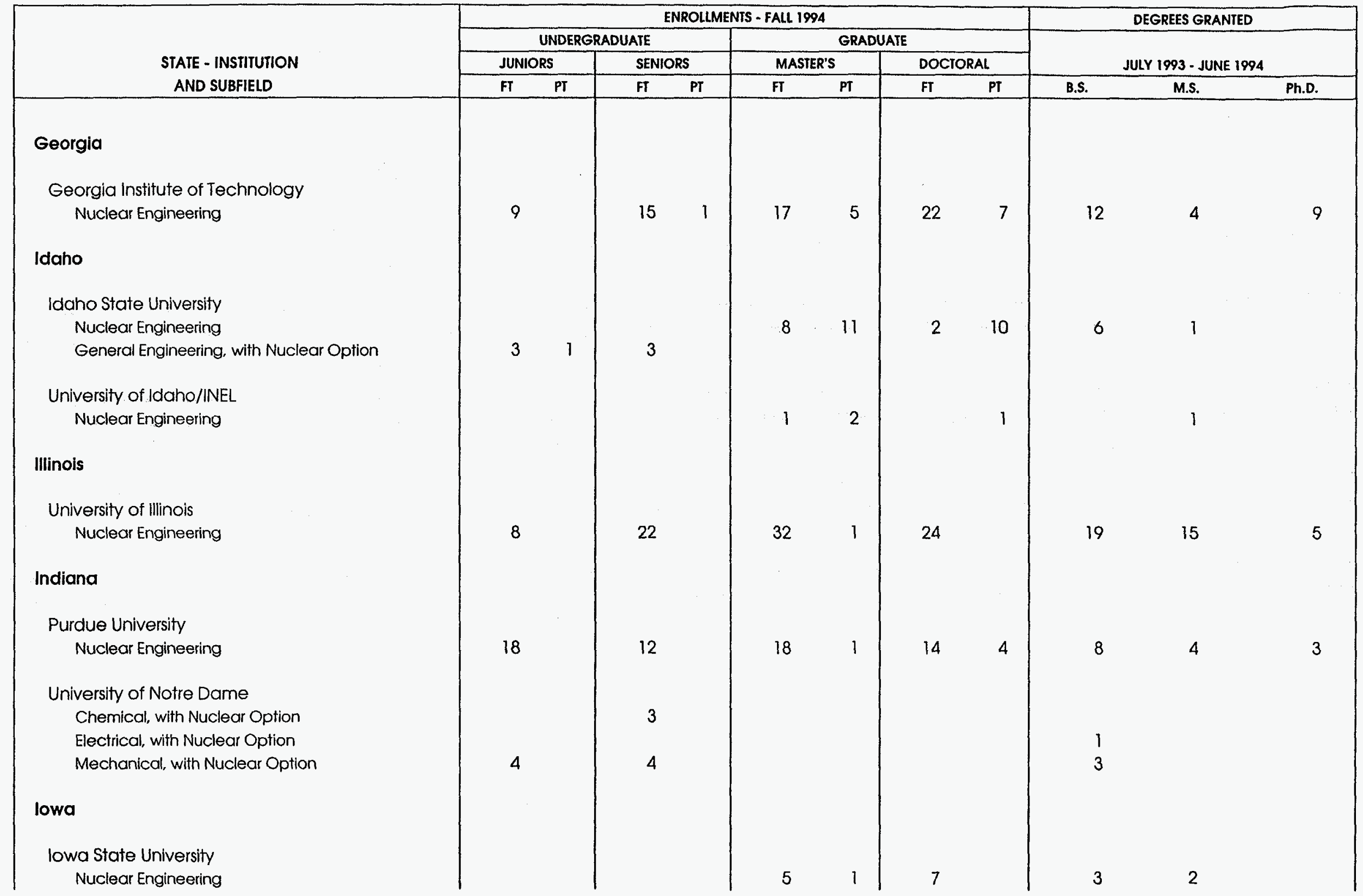




\section{1}

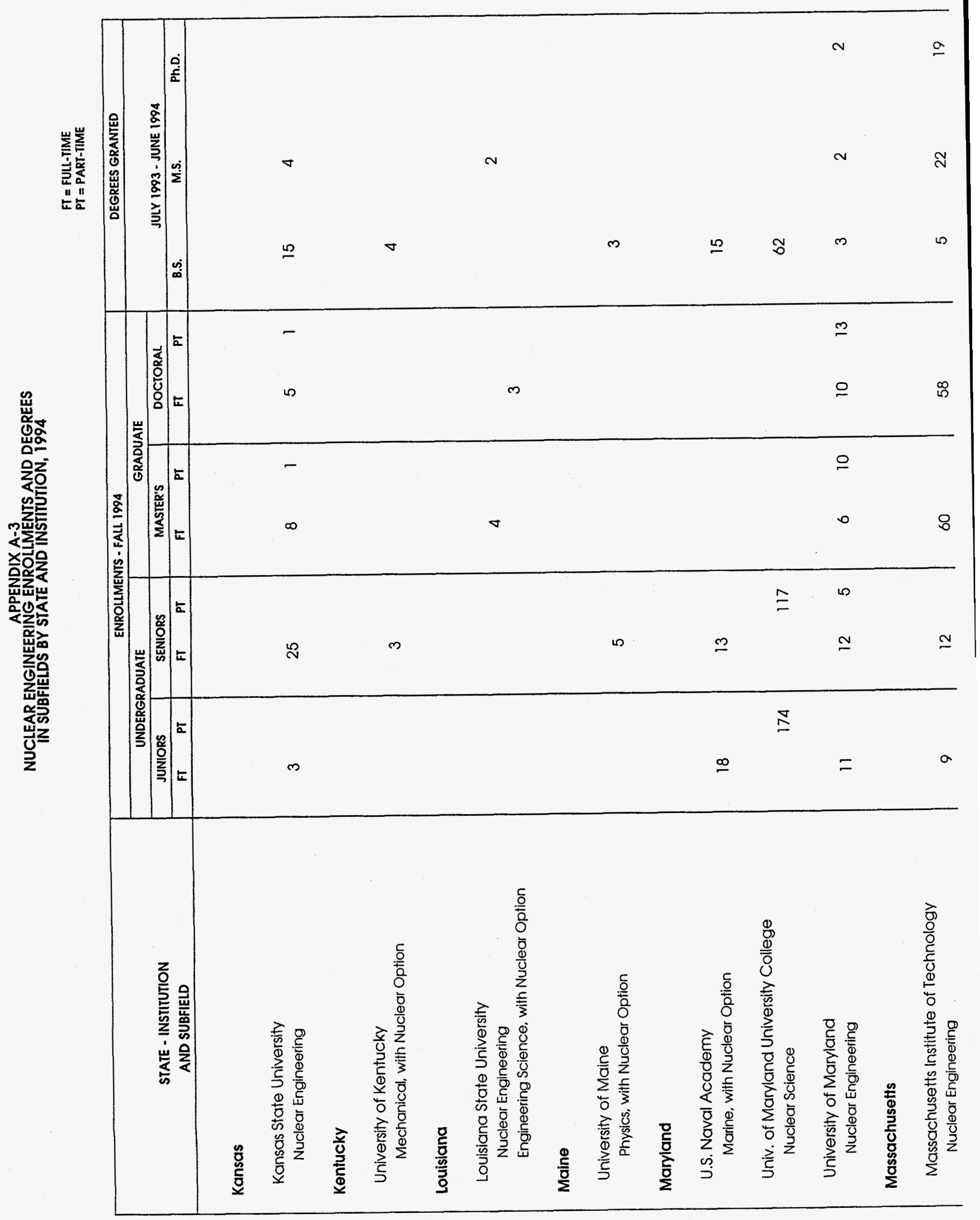


APPENDIX A-3

NUCLEAR ENGINEERNG ENROLMENTS AND DEGREES

IN SUBFIELDS BY STATE AND INSTITUTION, 1994

$F T=$ FULL-TIME

PI $=$ PART-TIME

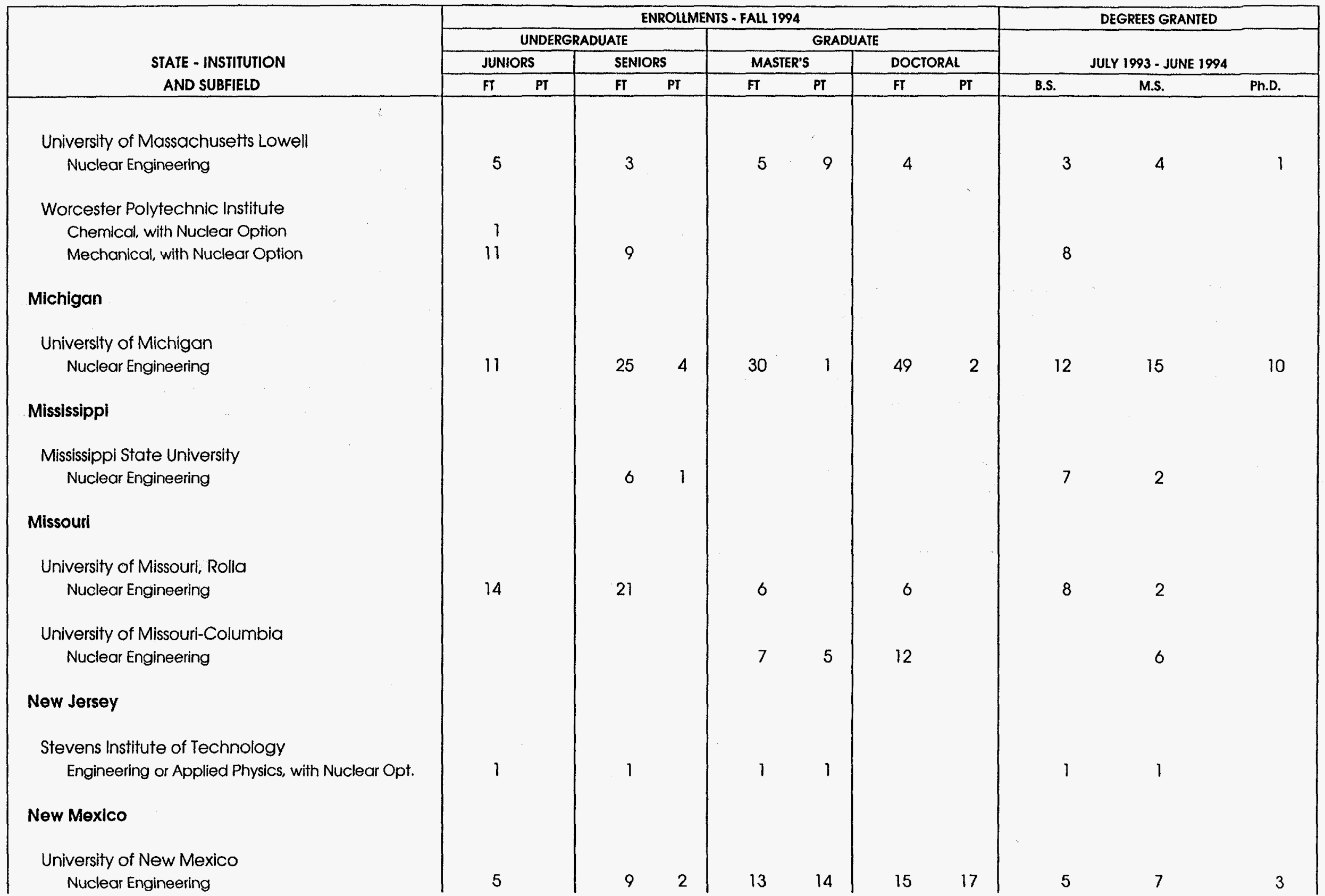


APPENDIX A-3

NUCLEAR ENGINEERING ENROAMENTS AND DEGREES

IN SUBFIELDS BY STATE AND INSTITUTION, 1994

FT = FULL-TIME

PT = PART-TIME

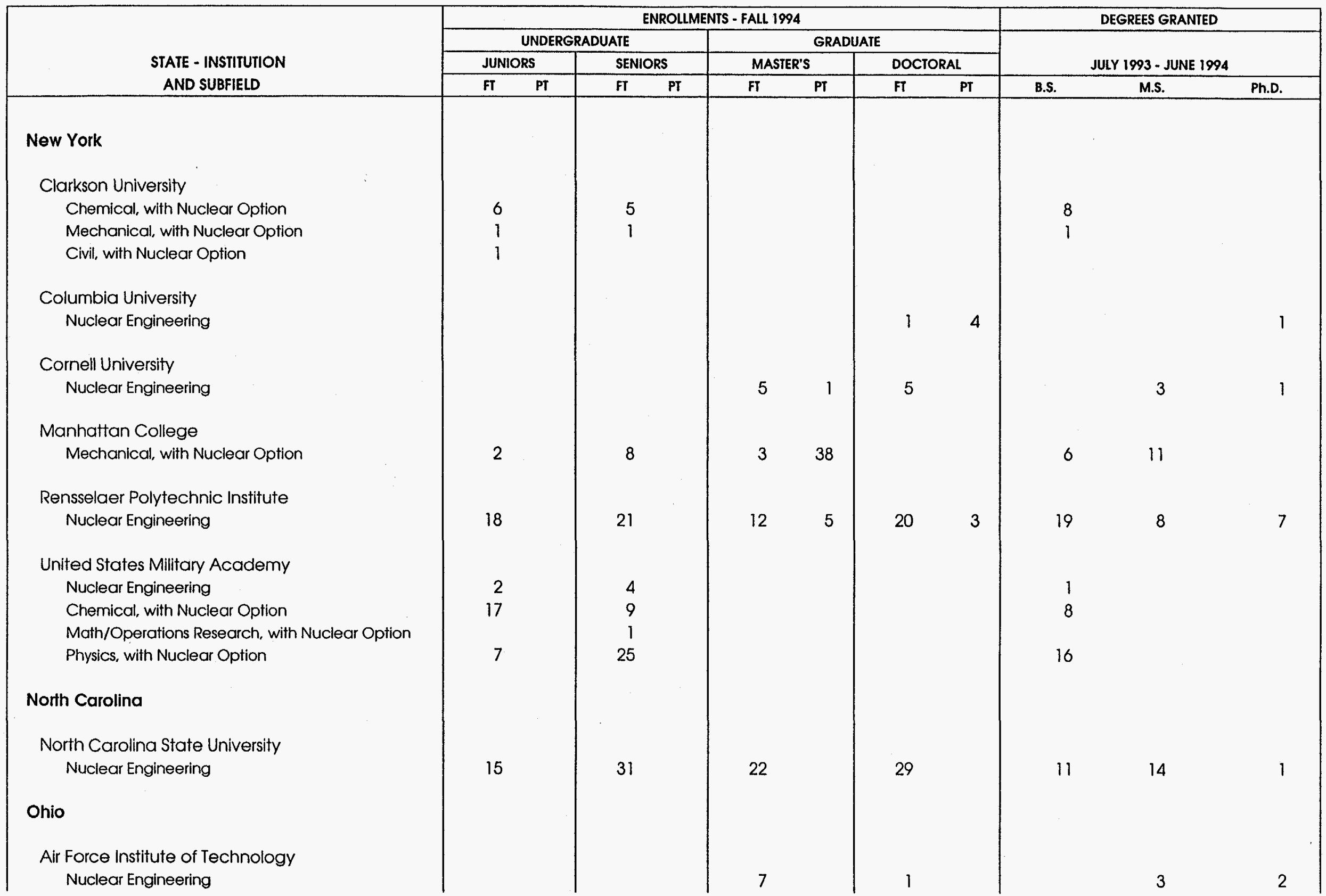




\section{APPENDIX A-3}

NUCLEAR ENGINEERING ENROLMENTS AND DEGREES

IN SUBFIELDS BY STATE AND INSTITUTION, 1994

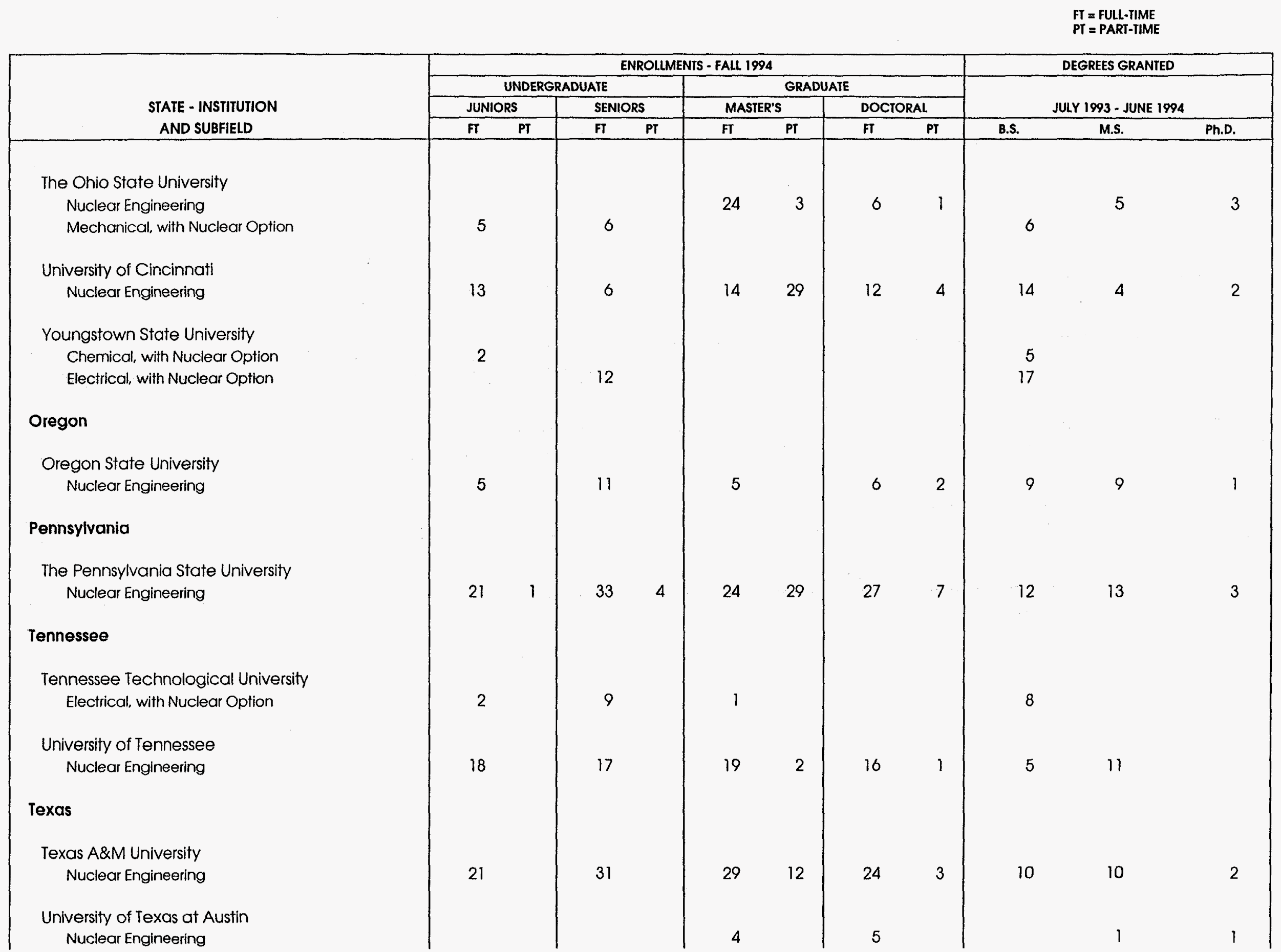


APPENDIX A-3

NUCLEAR ENGINEERING ENROLLMENTS AND DEGREES

IN SUBFIELDS BY STATE AND INSTITUTION, 1994

FT $=$ FULL-TIME

$\mathrm{PT}=\mathrm{PART}$

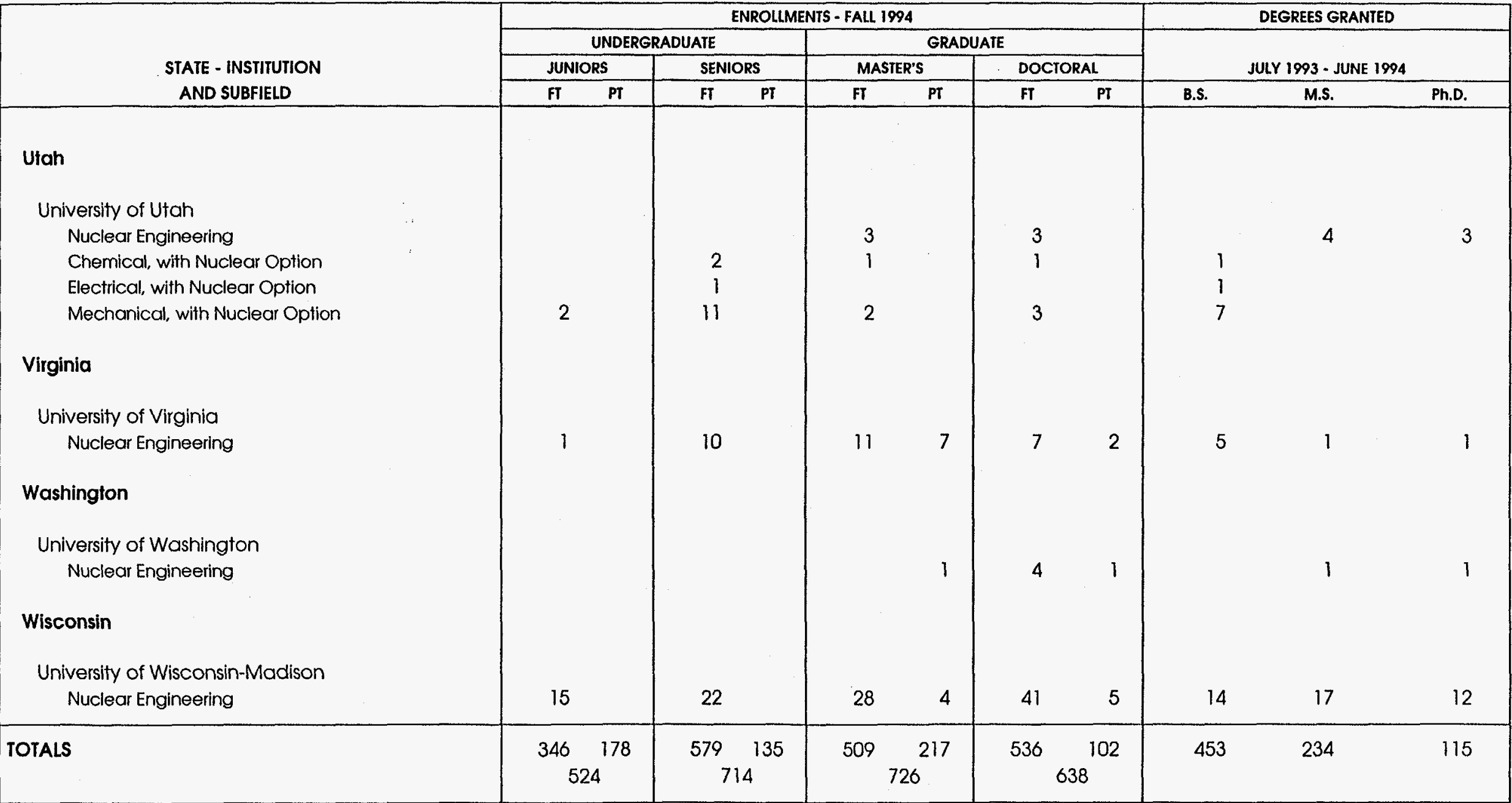

Source: U.S. Department of Energy 
NUCLEAR ENGINEERING ENROLLMENTS AND DEGREES BY SUBFIELD, 1994

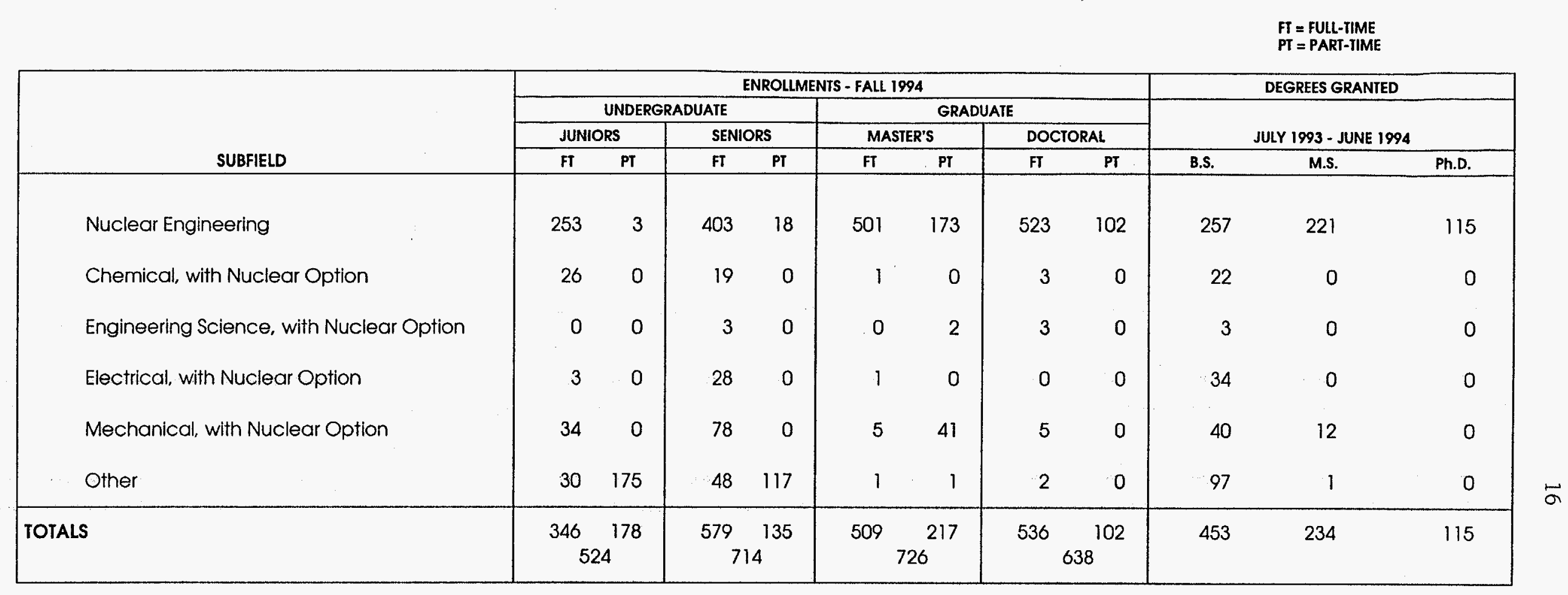

Source: U.S. Department of Energy 
APPENDIX A-5

UNDERGRADUATE, MASTER'S, AND DOCTORAL ENROLLMENTS AND DEGREES IN

NUCLEAR ENGINEERING, PERCENT DISTRIBUTION, 1990 - 1994

\begin{tabular}{|c|c|c|c|c|c|c|}
\hline \multirow[b]{3}{*}{ SUBFIELD } & \multicolumn{3}{|c|}{ ENROLLMENTS (Fall Term) } & \multicolumn{3}{|c|}{$\begin{array}{l}\text { PEGREES GRANIED } \\
\text { (Pear Ending June 30) } \\
\end{array}$} \\
\hline & UNDERGRADUATE & MASTER'S & DOCTORAL & B.S. & M.S. & PH.D. \\
\hline & $\%$ & $\%$ & $\%$ & $\%$ & $\%$ & $\%$ \\
\hline \multicolumn{7}{|l|}{ Nuclear Engineering } \\
\hline 1990 & 61.2 & 91.3 & 97.1 & 62.8 & 93.2 & 97.7 \\
\hline 1991 & 54.1 & 89.7 & 98.7 & 65.1 & 97.3 & 99.2 \\
\hline 1992 & 51.6 & 92.9 & 99.4 & 69.3 & 96.7 & 100.0 \\
\hline 1993 & 56.5 & 92.5 & 99.1 & 64.7 & 96.1 & 100.0 \\
\hline 1994 & 54.7 & 92.8 & 98.0 & 56.7 & 94.4 & 100.0 \\
\hline \multicolumn{7}{|c|}{ Chemical, with Nuclear Option } \\
\hline 1990 & 1.5 & 0.4 & 0.9 & 2.5 & 0.0 & 2.3 \\
\hline 1991 & 2.1 & 0.0 & 1.0 & 3.6 & 0.0 & 0.0 \\
\hline 1992 & 3.1 & 0.1 & 0.0 & 3.9 & 0.0 & 0.0 \\
\hline 1993 & 3.0 & 0.1 & 0.3 & 3.9 & 0.0 & 0.0 \\
\hline 1994 & 3.6 & 0.1 & 0.5 & 4.9 & 0.0 & 0.0 \\
\hline \multicolumn{7}{|c|}{ Engineering Sclence. with Nuclear Option } \\
\hline 1990 & 2.2 & 0.8 & 1.1 & 5.3 & 2.3 & 0.0 \\
\hline 1991 & 0.6 & 0.0 & 0.1 & 2.4 & 0.0 & 0.0 \\
\hline 1992 & 0.4 & 0.5 & 0.3 & 1.3 & 0.0 & 0.0 \\
\hline 1993 & 0.6 & 0.3 & 0.4 & 0.5 & 0.0 & 0.0 \\
\hline 1994 & 0.2 & 0.3 & 0.5 & 0.7 & 0.0 & 0.0 \\
\hline \multicolumn{7}{|c|}{ Electrical, with Nuclear Option } \\
\hline 1990 & 3.8 & 0.3 & 0.1 & 3.9 & 0.0 & 0.0 \\
\hline 1991 & 2.7 & 0.1 & 0.0 & 5.5 & 0.5 & 0.0 \\
\hline 1992 & 3.3 & 0.1 & 0.0 & 4.2 & 0.0 & 0.0 \\
\hline 1993 & 3.0 & 0.1 & 0.0 & 3.9 & 0.0 & 0.0 \\
\hline 1994 & 2.5 & 0.1 & 0.0 & 7.5 & 0.0 & 0.0 \\
\hline
\end{tabular}


APPENDIX A-5

UNDERGRADUATE, MASTER'S, AND DOCTORAL ENROLLMENTS AND DEGREES IN NUCLEAR ENGINEERING, PERCENT DISTRIBUTION, 1990 - 1994

\begin{tabular}{|c|c|c|c|c|c|c|}
\hline \multirow[b]{3}{*}{ SUBFIELD } & \multicolumn{3}{|c|}{ ENROLLMENTS (Fall Term) } & \multicolumn{3}{|c|}{$\begin{array}{l}\text { PEGREES GRANTEP } \\
\text { (rear Ending June 30) }\end{array}$} \\
\hline & UNDERGRADUATE & MASTER'S & DOCTORAL & B.S. & M.S. & PH.D. \\
\hline & $\%$ & $\%$ & $\%$ & $\%$ & $\%$ & $\%$ \\
\hline \multicolumn{7}{|c|}{ Mechanlcal, wilth Nuclear Option } \\
\hline 1990 & 9.2 & 4.8 & 0.4 & 14.9 & 2.3 & 0.0 \\
\hline 1991 & 7.0 & 7.4 & 0.1 & 10.3 & 0.5 & 0.8 \\
\hline 1992 & 9.3 & 6.3 & 0.3 & 8.3 & 3.3 & 0.0 \\
\hline 1993 & 7.7 & 6.7 & 0.1 & 11.2 & 3.9 & 0.0 \\
\hline 1994 & 9.0 & 6.3 & 0.8 & 8.8 & 5.1 & 0.0 \\
\hline \multicolumn{7}{|l|}{ Other } \\
\hline 1990 & 22.1 & 2.4 & 0.3 & 10.6 & 2.3 & 0.0 \\
\hline 1991 & 30.9 & 2.8 & 0.0 & 13.2 & 1.8 & 0.0 \\
\hline 1992 & 32.3 & 0.1 & 0.0 & 13.0 & 0.0 & 0.0 \\
\hline 1993 & 29.2 & 0.3 & 0.0 & 15.8 & 0.0 & 0.0 \\
\hline 1994 & 29.9 & 0.3 & 0.3 & 21.4 & 0.4 & 0.0 \\
\hline
\end{tabular}

Source: U.S. Department of Energy 
APPENDIX A-6

FOREIGN NATIONAL, FEMALE, AND MINORITY PARTICIPATION

IN NUCLEAR ENGINEERING PROGRAMS, 1988 - 1994

\begin{tabular}{|c|c|c|c|c|c|c|c|c|c|c|c|c|c|c|}
\hline \multirow{3}{*}{$\begin{array}{l}\text { EXHNIC GROUP } \\
\text { AND GENDER }\end{array}$} & \multicolumn{8}{|c|}{ ENROLLMENTS (Fall Term) } & \multicolumn{6}{|c|}{$\begin{array}{l}\text { PEGREES GRANIED } \\
\text { (Year Ending une } 30 \text { ) }\end{array}$} \\
\hline & \multicolumn{2}{|c|}{ JUNIORS } & \multicolumn{2}{|c|}{ SENIORS } & \multicolumn{2}{|c|}{ MASIER'S } & \multicolumn{2}{|c|}{ DOCTORAL } & \multicolumn{2}{|c|}{ B.S. } & \multicolumn{2}{|c|}{ M.S. } & \multicolumn{2}{|c|}{ PH.D. } \\
\hline & No. & $\%$ & NO. & $\%$ & No. & $\%$ & NO. & $\%$ & No. & $\%$ & No. & $\%$ & No. & $\%$ \\
\hline \multicolumn{15}{|l|}{ Foreign Nationals } \\
\hline 1988 & 13 & 2 & 14 & 2 & 207 & 28 & 332 & 51 & 21 & 4 & 87 & 38 & 56 & 49 \\
\hline 1989 & 14. & 2 & 17 & 2 & 218 & 29 & 336 & 50 & 6 & 1 & 69 & 24 & 46 & 45 \\
\hline 1990 & 15 & 2 & 20 & 3 & 197 & 27 & 360 & 51 & 10 & 2 & 62 & 28 & 65 & 49 \\
\hline 1991 & 9 & 1 & 19 & 3 & 207 & 26 & 332 & 47 & 9 & 2 & 69 & 31 & 55 & 45 \\
\hline 1992 & 16 & 2 & 15 & 2 & 187 & 23 & 326 & 50 & 15 & 4 & 65 & 31 & 63 & 47 \\
\hline 1993 & 20 & 3 & 22 & 3 & 185 & 24 & 353 & 51 & 10 & 2 & 74 & 29 & 67 & 55 \\
\hline 1994 & 12 & 2 & 41 & 6 & 142 & 20 & 256 & 40 & 13 & 3 & 55 & 24 & 60 & 52 \\
\hline \multicolumn{15}{|l|}{ Women } \\
\hline 1988 & 61 & 9 & 69 & 10 & 78 & 10 & 56 & 9 & 51 & 11 & 21 & 9 & 0 & 5 \\
\hline 1989 & 60 & 8 & 73 & 10 & 85 & 11 & 53 & 8 & 45 & 10 & 30 & 10 & 7 & 7 \\
\hline 1990 & 67 & 9 & 81 & 11 & 85 & 12 & 63 & 9 & 48 & 11 & 23 & 10 & 10 & 8 \\
\hline 1991 & 55 & 7 & 68 & 10 & 99 & 13 & 62 & 9 & 58 & 14 & 22 & 10 & 8 & 7 \\
\hline 1992 & 64 & 8 & 65 & 9 & 110 & 13 & 58 & 9 & 39 & 10 & 34 & 16 & 9 & 7 \\
\hline 1993 & 64 & 9 & 52 & 7 & 99 & 13 & 71 & 10 & 41 & 10 & 33 & 13 & 13 & 11 \\
\hline 1994 & 51 & 10 & 61 & 9 & 80 & 11 & 67 & 11 & 36 & 8 & 26 & 11 & 14 & 12 \\
\hline \multicolumn{15}{|l|}{ Blacks } \\
\hline 1988 & 26 & 4 & 12 & 2 & 8 & 1 & 2 & 0 & 5 & 1 & 1 & 0 & 2 & 2 \\
\hline 1989 & 24 & 3 & 11 & 2 & 6 & 1 & 4 & 1 & 5 & 1 & 2 & 1 & 0 & 0 \\
\hline 1990 & 13 & 2 & 20 & 3 & 5 & 1 & 2 & 0 & 8 & 2 & 2 & 1 & 0 & 0 \\
\hline 1991 & 9 & 1 & 21 & 3 & 11 & 1 & 2 & 0 & 10 & 2 & 1 & 0 & 0 & 0 \\
\hline 1992 & 20 & 3 & 24 & 3 & 11 & 1 & 5 & 1 & 4 & 1 & 0 & 0 & 0 & 0 \\
\hline 1993 & 22 & 3 & 19 & 3 & 11 & 1 & 9 & 1 & 11 & 3 & 5 & 2 & 5 & 4 \\
\hline 1994 & 10 & 2 & 15 & 2 & 13 & 2 & 6 & 1 & 12 & 3 & 2 & 1 & 0 & 0 \\
\hline
\end{tabular}


APPENDIX A-6

FOREIGN NATIONAL, FEMALE, AND MINORITY PARTICIPATION

IN NUCLEAR ENGINEERING PROGRAMS, 1988 - 1994

\begin{tabular}{|c|c|c|c|c|c|c|c|c|c|c|c|c|c|c|}
\hline \multirow{3}{*}{$\begin{array}{l}\text { ETHNIC GROUP } \\
\text { AND GENDER }\end{array}$} & \multicolumn{8}{|c|}{ ENROLLMENTS (Fall Term) } & \multicolumn{6}{|c|}{$\begin{array}{l}\text { PEGREES GRANIEP } \\
\text { (Year Ending June 30) }\end{array}$} \\
\hline & \multicolumn{2}{|c|}{ JUNIORS } & \multicolumn{2}{|c|}{ SENIORS } & \multicolumn{2}{|c|}{ MASTER'S } & \multicolumn{2}{|c|}{ DOCTORAL } & \multicolumn{2}{|c|}{ B.S. } & \multicolumn{2}{|c|}{ M.S. } & \multicolumn{2}{|c|}{ PH.D. } \\
\hline & NO. & $\%$ & No. & $\%$ & NO. & $\%$ & No. & $\%$ & NO. & $\%$ & No. & $\%$ & NO. & $\%$ \\
\hline \multicolumn{15}{|l|}{ Hispanic U.S. Citizens } \\
\hline 1988 & 12 & 2 & 19 & 3 & 10 & 1 & 6 & 1 & 5 & 1 & 1 & 0 & 0 & 0 \\
\hline 1989 & 10 & 1 & 14 & 2 & 12 & 2 & 8 & 1 & 11 & 2 & 3 & 1 & 1 & 1 \\
\hline 1990 & 23 & 3 & 13 & 2 & 17 & 2 & 9 & 1 & 4 & 1 & 1 & 0 & 3 & 2 \\
\hline 1991 & 11 & 1 & 20 & 3 & 12 & 2 & 8 & 1 & 8 & 2 & 5 & 2 & 0 & 0 \\
\hline 1992 & 16 & 2 & 20 & 3 & 11 & 1 & 16 & 2 & 9 & 2 & 2 & 1 & 0 & 0 \\
\hline 1993 & 18 & 3 & 16 & 2 & 19 & 2 & 14 & 2 & 7 & 2 & 6 & 2 & 5 & 4 \\
\hline 1994 & 13 & 2 & 11 & 2 & 18 & 2 & 19 & 3 & 9 & 2 & 3 & 1 & 1 & 1 \\
\hline \multicolumn{15}{|c|}{ Asian Americans/Pacific Islanders } \\
\hline 1988 & 20 & 3 & 24 & 3 & 27 & 4 & 25 & 4 & 13 & 3 & 9 & 4 & 3 & 3 \\
\hline 1989 & 6 & 1 & 21 & 3 & 16 & 2 & 24 & 4 & 7 & 2 & 9 & 3 & 1 & 1 \\
\hline 1990 & 15 & 2 & 21 & 3 & 8 & 1 & 25 & 4 & 23 & 5 & 4 & 2 & 3 & 2 \\
\hline 1991 & 25 & 3 & 24 & 3 & 14 & 2 & 20 & 3 & 13 & 3 & 5 & 2 & 9 & 7 \\
\hline 1992 & 24 & 3 & 27 & 4 & 14 & 2 & 21 & 3 & 13 & 3 & 9 & 4 & 3 & 2 \\
\hline 1993 & 25 & 4 & 25 & 4 & 14 & 2 & 21 & 3 & 7 & 2 & 6 & 2 & 6 & 5 \\
\hline 1994 & 11 & 2 & 24 & 3 & 30 & 4 & 48 & 8 & 15 & 3 & 5 & 2 & 4 & 3 \\
\hline \multicolumn{15}{|c|}{ American Indians/Alaskan Natives } \\
\hline 1988 & 0 & 0 & 0 & 0 & 2 & 0 & 1 & 0 & 1 & 0 & 0 & 0 & 0 & 0 \\
\hline 1989 & 1 & 0 & 0 & 0 & 1 & 0 & 1 & 0 & 3 & 1 & 1 & 0 & 0 & 0 \\
\hline 1990 & 0 & 0 & 2 & 0 & 1 & 0 & 2 & 0 & 0 & 0 & 1 & 0 & 0 & 0 \\
\hline 1991 & 1 & 0 & 2 & 0 & 0 & 0 & 2 & 0 & 0 & 0 & 0 & 0 & 0 & 0 \\
\hline 1992 & 2 & 0 & 2 & 0 & 0 & 0 & 1 & 0 & 1 & 0 & 0 & 0 & 0 & 0 \\
\hline 1993 & 3 & 0 & 2 & 0 & 1 & 0 & 1 & 0 & 1 & 0 & 1 & 0 & 0 & 0 \\
\hline 1994 & 0 & 0 & 4 & 1 & 0 & 0 & 1 & 0 & 1 & 0 & 0 & 0 & 0 & 0 \\
\hline
\end{tabular}

NOTE: All zeros in the table represent values of less than 0.5 percent.

Source: U.S. Department of Energy 
APPENDIXA-7

NUCLEAR ENGINEERING: FOREIGN NATIONAL FEMALE AND MINORITY

ENROLLMENTS AND DEGREES BY STATE AND INSTITUTION, 1994

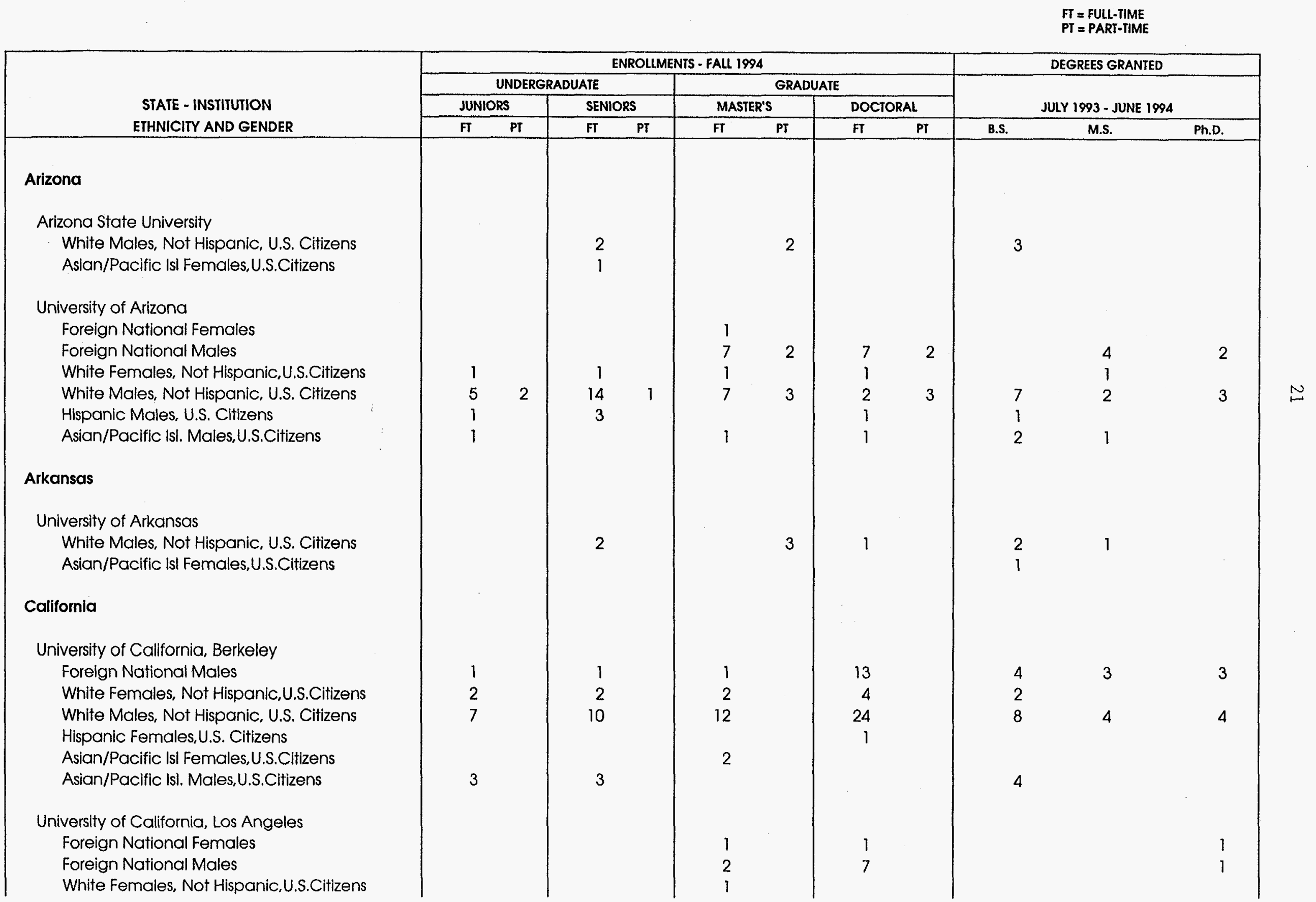


APPENDIX A-7

NUCLEAR ENGINEERING: FOREIGN NATIONAL, FEMALE, AND MINORITY

ENROLLMENIS AND DEGREES BY STATE AND INSTITUTION, 1994

FT = FULL-TIME

PT = PART-TIME

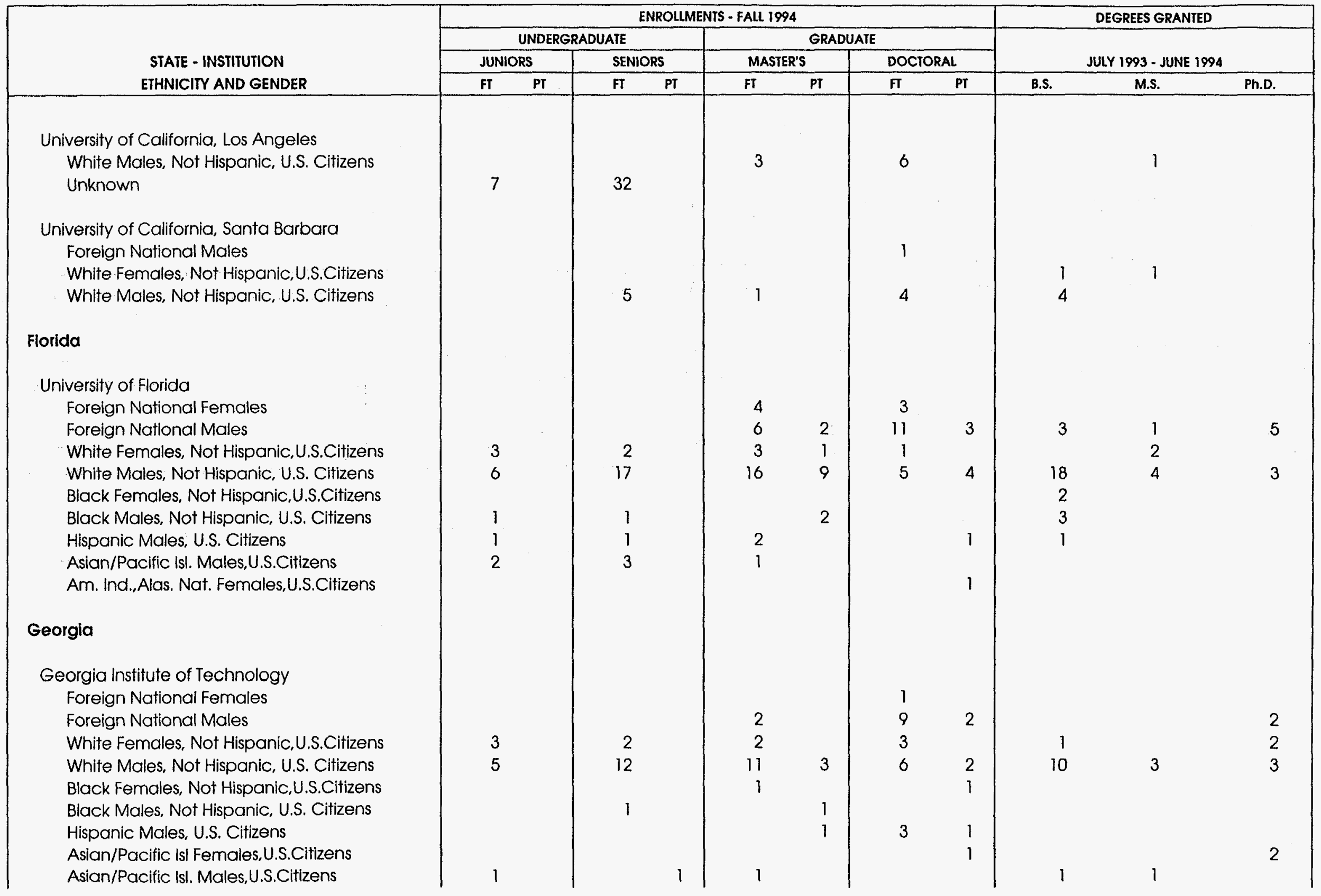


APPENDIX A-7

NUCLEAR ENGINEERING: FOREIGN NATIONAL FEMALE AND MINORITY

ENROLLMENTS AND DEGREES BY STATE AND INSTITUTION, 1994

$F I=F U L L-T I M E$

PT $=$ PART-TIME

\begin{tabular}{|c|c|c|c|c|c|c|c|c|c|c|c|}
\hline \multirow{4}{*}{$\begin{array}{l}\text { STATE - INSTITUTION } \\
\text { ETHNICITY AND GENDER }\end{array}$} & \multicolumn{8}{|c|}{ ENROLLMENTS - FALL 1994} & \multicolumn{3}{|c|}{ DEGREES GRANTED } \\
\hline & \multicolumn{4}{|c|}{ UNDERGRADUAIE } & \multicolumn{4}{|c|}{ GRADUAIE } & & & \\
\hline & \multicolumn{2}{|c|}{ JUNIORS } & \multicolumn{2}{|c|}{ SENIORS } & \multicolumn{2}{|c|}{ MASTER'S } & \multicolumn{2}{|c|}{ DOCTORAL } & \multicolumn{3}{|c|}{ JULY 1993 - JUNE 1994} \\
\hline & FT & PI & FT & PT & FT & PT & FT & PT & B.S. & M.S. & Ph.D. \\
\hline \multicolumn{12}{|l|}{ Idaho } \\
\hline \multicolumn{12}{|l|}{ Idaho State University } \\
\hline Foreign National Males & 2 & & & & 1 & & 2 & & & & \\
\hline White Females, Not Hispanic. U.S.Citizens & & & & & & 1 & & & 2 & & \\
\hline White Males, Not Hispanic, U.S. Citizens & 1 & & 3 & & 7 & 10 & & 10 & 4 & 1 & \\
\hline Asian/Pacific |s|. Males, U.S.Citizens & & 1 & & & & & & & & & \\
\hline \multicolumn{12}{|l|}{ University of Idaho/INEL. } \\
\hline White Males, Not Hispanic. U.S. Citizens & & & & & 1 & 2 & & 1 & & 1 & \\
\hline \multicolumn{12}{|l|}{ Illinois } \\
\hline \multicolumn{12}{|l|}{ University of Illinois } \\
\hline Foreign National Females & & & & & 5 & & 3 & & & & 2 \\
\hline Forelgn National Males & & & & & 1 & & 8 & & & 4 & 2 \\
\hline White Females, Not Hispanic, U.S.Citizens & & & 2 & & 2 & & 2 & & 2 & 1 & \\
\hline White Males, Not Hispanic, U.S. Citizens & 7 & & 19 & & 23 & 1 & 10 & & 16 & 10 & 1 \\
\hline Black Males, Not Hispanic, U.S. Citizens & & & & & & & & & 1 & & \\
\hline Hispanic Males, U.S. Citizens & 1 & & 1 & & 1 & & 1 & & & & \\
\hline \multicolumn{12}{|l|}{ Indiana } \\
\hline \multicolumn{12}{|l|}{ Purdue University } \\
\hline Foreign National Females & & & & & & & & & & & 1 \\
\hline Foreign National Females & & & & & & & & & & & \\
\hline Foreign National Males & & & & & 9 & 1 & 12 & 1 & & 1 & 2 \\
\hline White Females, Not Hispanic.U.S.Citizens & 4 & & & & & & & & 1 & 1 & \\
\hline White Males, Not Hispanic, U.S. Citizens & 13 & & 11 & & 7 & & & 3 & 6 & 2 & \\
\hline Hispanic Females, U.S. Citizens & & & & & 1 & & & & 1 & & \\
\hline Hispanic Males, U.S. Citizens & 1 & & & & & & 1 & & & & \\
\hline Asian/Pacific IsI. Males.U.S.Citizens & & & 1 & & 1 & & 1 & & & & \\
\hline
\end{tabular}


APPENDIX A-7

NUCLEAR ENGINEERING: FOREIGN NATIONAL, FEMALE, AND MINORITY

ENROLLMENTS AND DEGREES BY STATE AND INSTIFUTION, 1994

$\mathrm{FT}=$ FULL-TIME

PT = PART-TIME

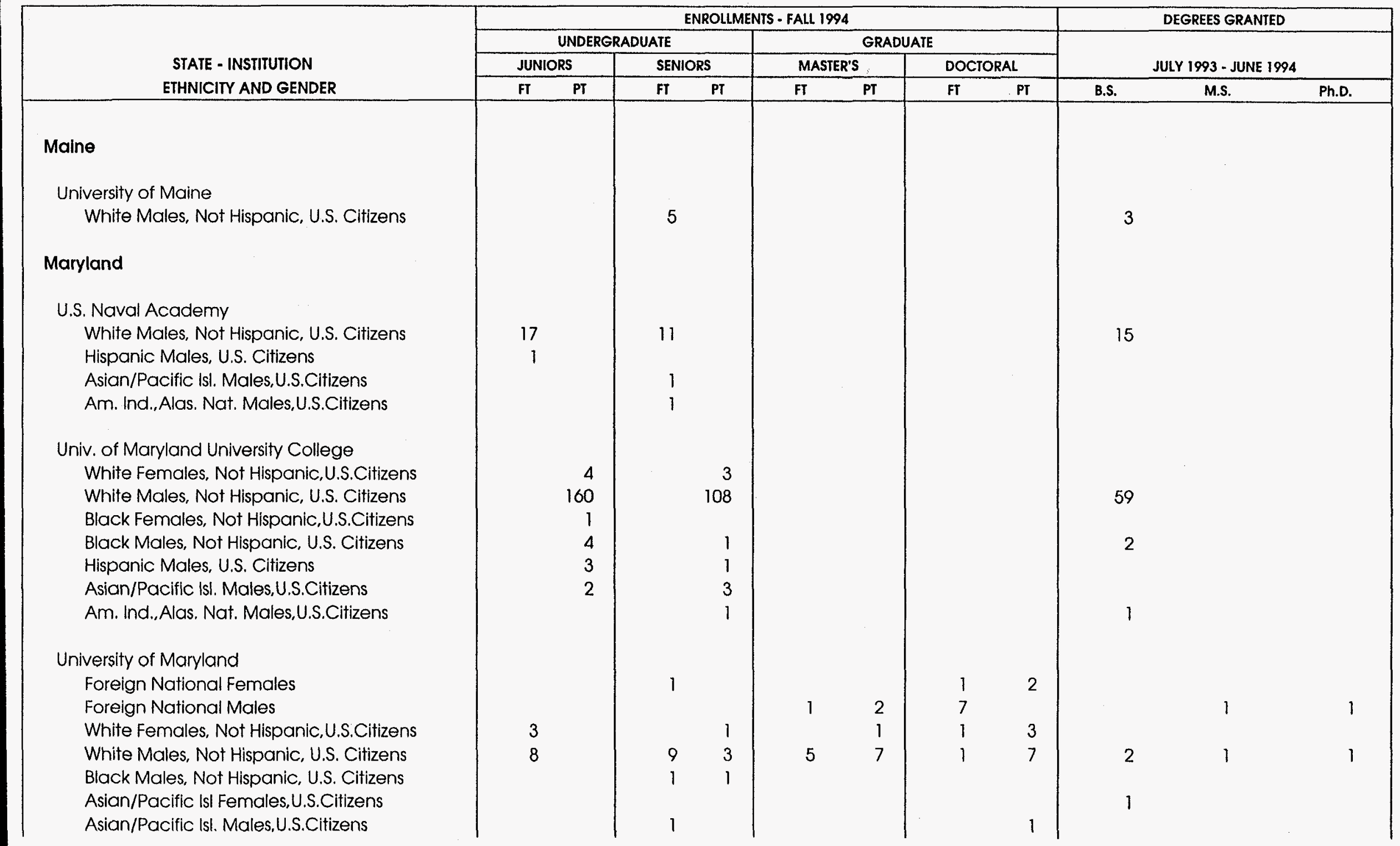


APPENDIX A-7

NUCLEAR ENGINEERING: FOREIGN NATIONAL. FEMALE AND MINORITY

ENROLLMENTS AND DEGREES BY STATE AND INSTITUTION, 1994

FT $=$ FULL - TIME

PT $=$ PART-TIME

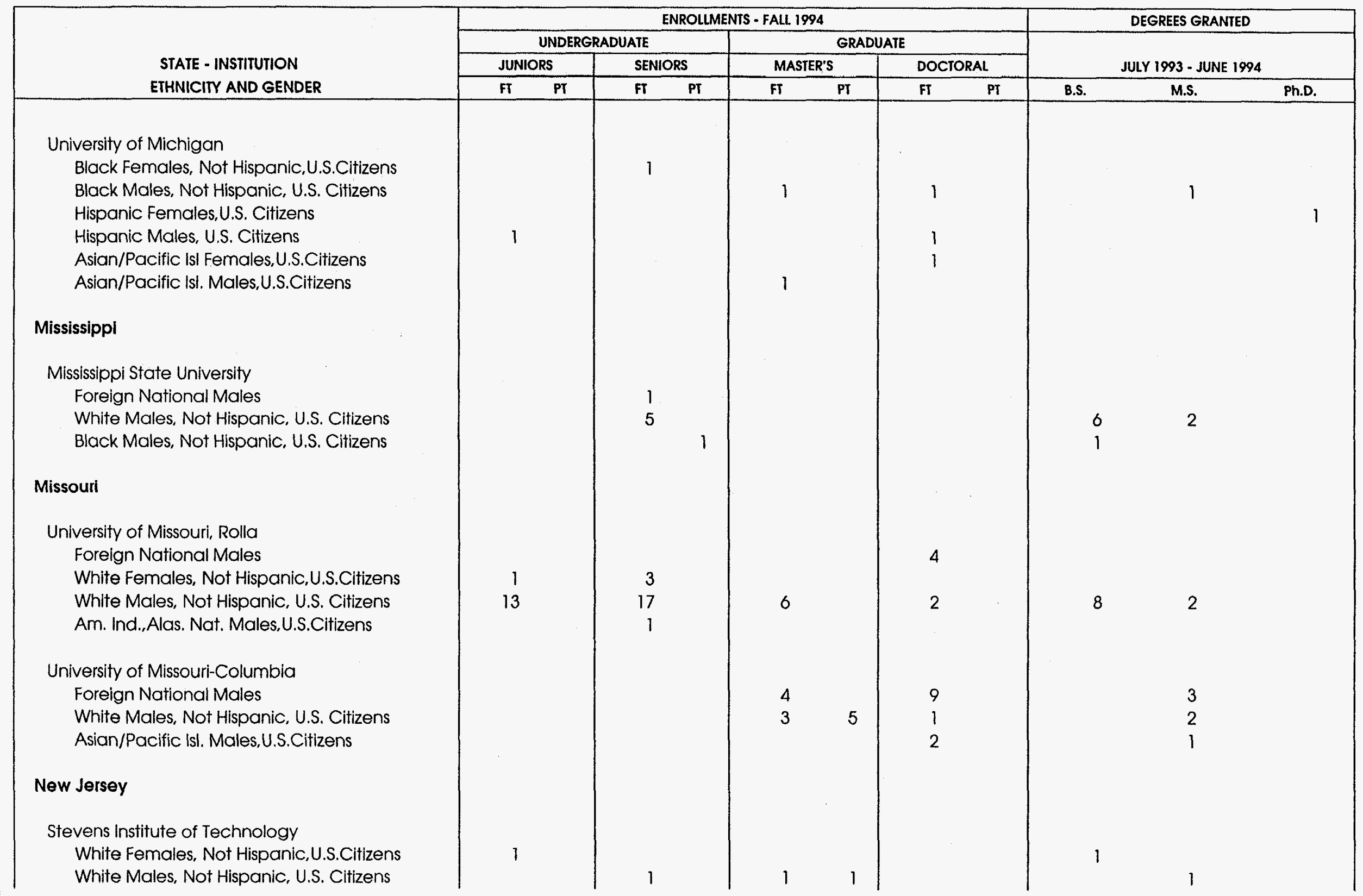


APPENDIX A-7

NUCLEAR ENGINEERING: FOREIGN NATIONAL FEMALE AND MINORITY

ENROLLMENTS AND DEGREES BY STATE AND INSTITUTION, 1994

FT $=$ FULL-TIME

PT $=$ PART - TIME

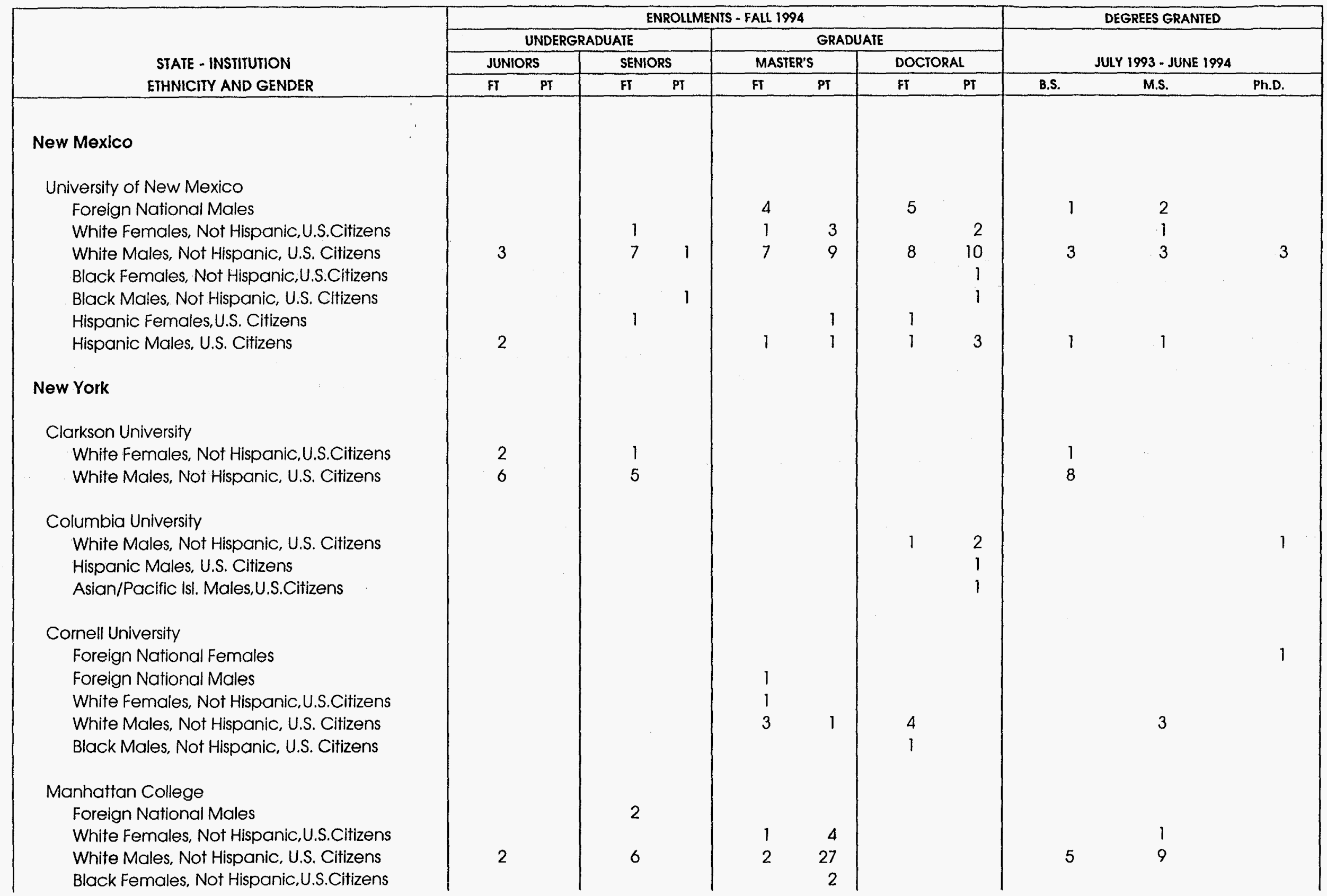


APPENDIX A-7

NUCLEAR ENGINEERING: FOREIGN NATIONA FEMALE, AND MINORITY

ENROLLMENTS AND DEGREES BY STATE AND INSTITUTION, 1994

$F I=$ FULL-TIME

PT $=$ PART-TIME

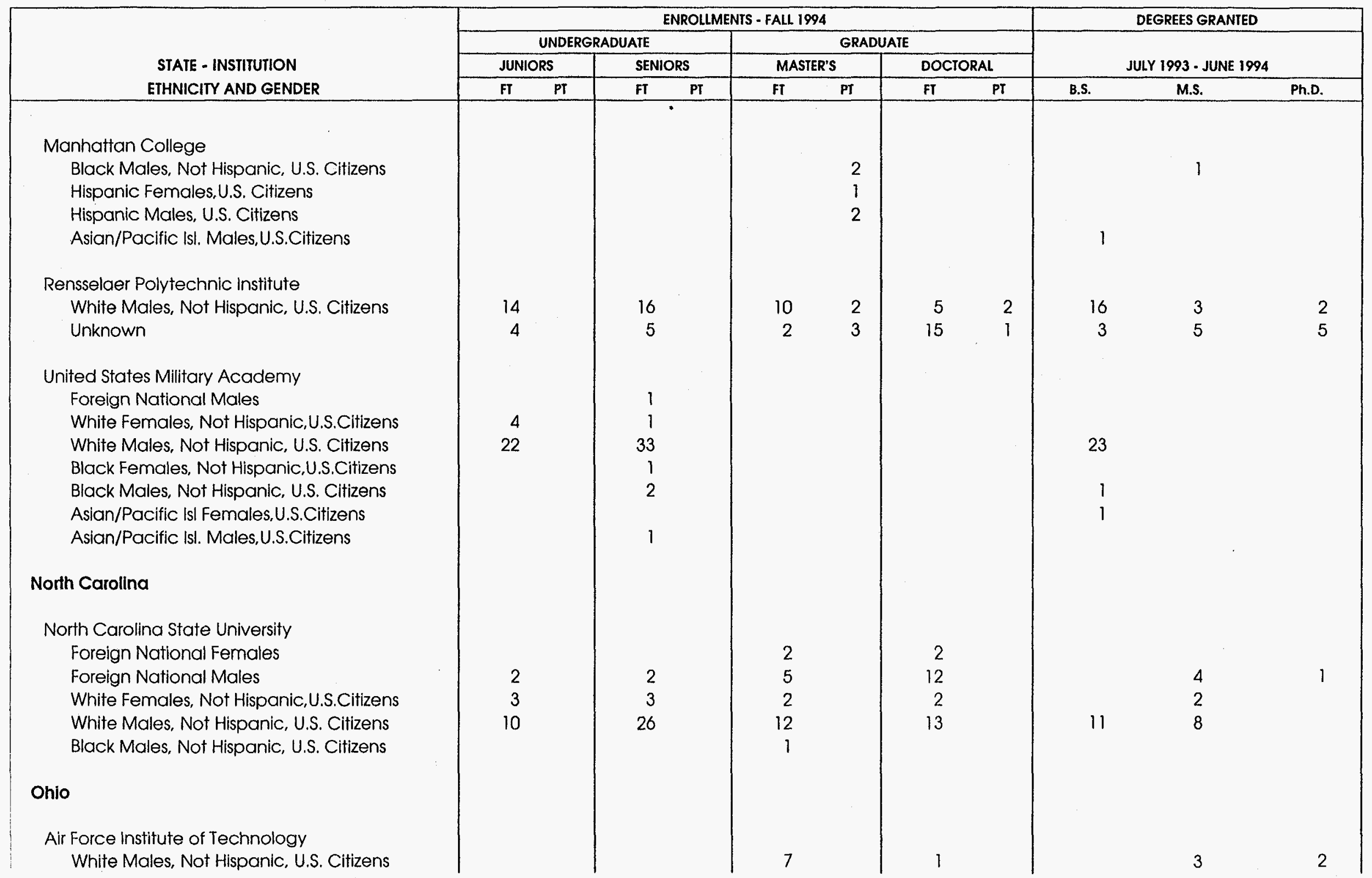


APPENDIX A-7

NUCLEAR ENGINEERING: FOREIGN NATIONAL, FEMALE, AND MINORITY

ENROLLMENTS AND DEGREES BY STATE AND INSTITUTION, 1994

$F T=F U L L-T I M E$

PI $=$ PART-TIME

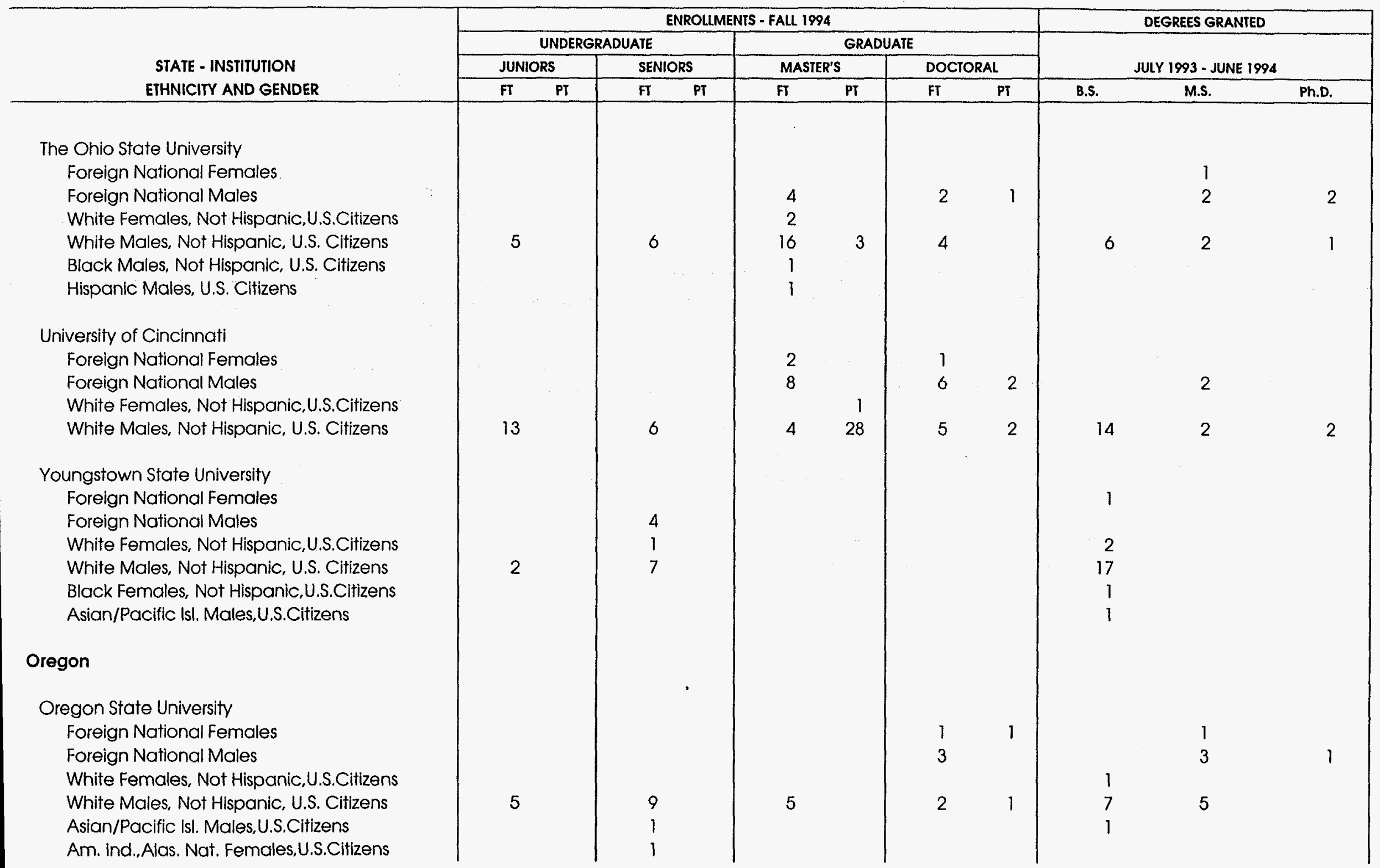


APPENDIX A-7

NUCLEAR ENGINEERING: FOREIGN NATIONAL FEMALE AND MINORITY

ENROLLMENTS AND DEGREES BY STATE AND INSTITUTION, 1994

$\mathrm{FT}=$ FULL-TIME

PT $=$ PART-TIME

\begin{tabular}{|c|c|c|c|c|c|c|c|c|c|c|c|}
\hline \multirow{4}{*}{$\begin{array}{l}\text { STATE - INSTITUTION } \\
\text { ETHNICITY AND GENDER }\end{array}$} & \multicolumn{8}{|c|}{ ENROLLMENTS - FALL 1994} & \multicolumn{3}{|c|}{ DEGREES GRANIED } \\
\hline & \multicolumn{4}{|c|}{ UNDERGRADUATE } & \multicolumn{4}{|c|}{ GRADUATE } & & & \\
\hline & \multicolumn{2}{|c|}{ JUNIORS } & \multicolumn{2}{|c|}{ SENIORS } & \multicolumn{2}{|c|}{ MASTER'S } & \multicolumn{2}{|c|}{ DOCIORAL } & & $993-\mathrm{J}$ & \\
\hline & $\mathbf{F T}$ & PT & FT & PT & FT & PT & FT & PT & B.S. & M.S. & Ph.D. \\
\hline \multicolumn{12}{|l|}{ Pennsylvania } \\
\hline The Pennsylvania State University & & & & & & & & & & & \\
\hline Foreign National Females & & & & & & & 1 & 1 & & & 1 \\
\hline Foreign National Males & & & & & 6 & & 13 & 1 & & 3 & 2 \\
\hline White Females, Not Hispanic, U.S.Citizens & 2 & & 5 & & 1 & 3 & 1 & & 3 & 1 & \\
\hline White Males, Not Hispanic, U.S. Citizens & 18 & 1 & 28 & 3 & 16 & 24 & 11 & 4 & 9 & 9 & \\
\hline Black Males, Not Hispanic, U.S. Citizens & & & & & 1 & & & & & & \\
\hline Hispanic Males, U.S. Citizens & 1 & & & & & 2 & & & & & \\
\hline Asian/Pacific Isl. Males, U.S.Citizens & & & & 1 & & & 1 & 1 & & & \\
\hline \multicolumn{12}{|l|}{ Tennessee } \\
\hline Tennessee Technological University & & & & & & & & & & & \\
\hline Foreign National Males & & & 1 & & & & & & & & \\
\hline White Females, Not Hispanic, U.S.Citizens & & & 1 & & & & & & & & \\
\hline White Males, Not Hispanic, U.S. Citizens & 1 & & 7 & & 1 & & & & 8 & & \\
\hline Black Males, Not Hispanic, U.S. Citizens & 1 & & & & & & & & & & \\
\hline \multicolumn{12}{|l|}{ University of Tennessee } \\
\hline Foreign National Females & & & & & 1 & & & & & & \\
\hline Foreign National Males & & & 1 & & 7 & 1 & 13 & & & 1 & \\
\hline White Females, Not Hispanic, U.S.Citizens & 5 & & 2 & & & & & & & & \\
\hline White Males, Not Hispanic, U.S. Citizens & 12 & & 12 & & 10 & 1 & 3 & 1 & 4 & 10 & \\
\hline Black Females, Not Hispanic, U.S.Citizens & & & 1 & & & & & & & & \\
\hline Black Males, Not Hispanic, U.S. Citizens & 1 & & 1 & & & & & & & & \\
\hline Asian/Pacific Isl. Males, U.S.Citizens & & & & & 1 & & & & 1 & & \\
\hline \multicolumn{12}{|l|}{ Texas } \\
\hline Texas A\&M University & & & & & & & & & & & \\
\hline Foreign National Females & & & 1 & & 2 & 1 & 2 & & & & \\
\hline Foreign National Males & 1 & & 2 & & 9 & 1 & 12 & & 1 & 2 & 2 \\
\hline White Females, Not Hispanic, U.S.Citizens & 2 & & 2 & & & & & & & 2 & \\
\hline
\end{tabular}


NUCLEAR ENGINEERING: FOREIGENDIX A-7

ENROLLMENTS AND DEGREES BY STATE ANND INSTITUTION, 1994

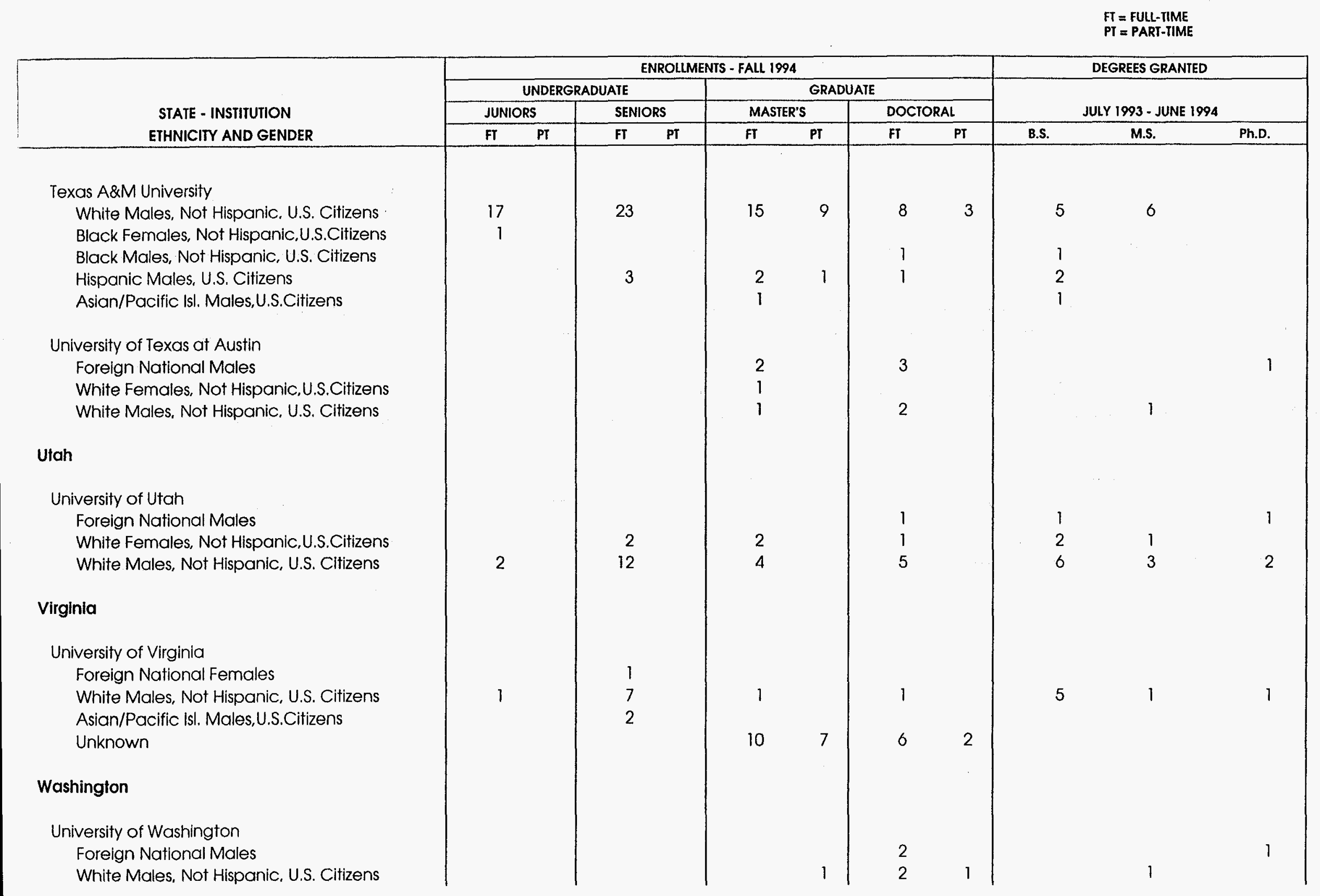


APPENDIX A-7

NUCLEAR ENGINEERING: FOREIGN NATIONAL FEMALE AND MINORITY

ENROLLMENTS AND DEGREES BY STATE AND INSTITUTION, 1994

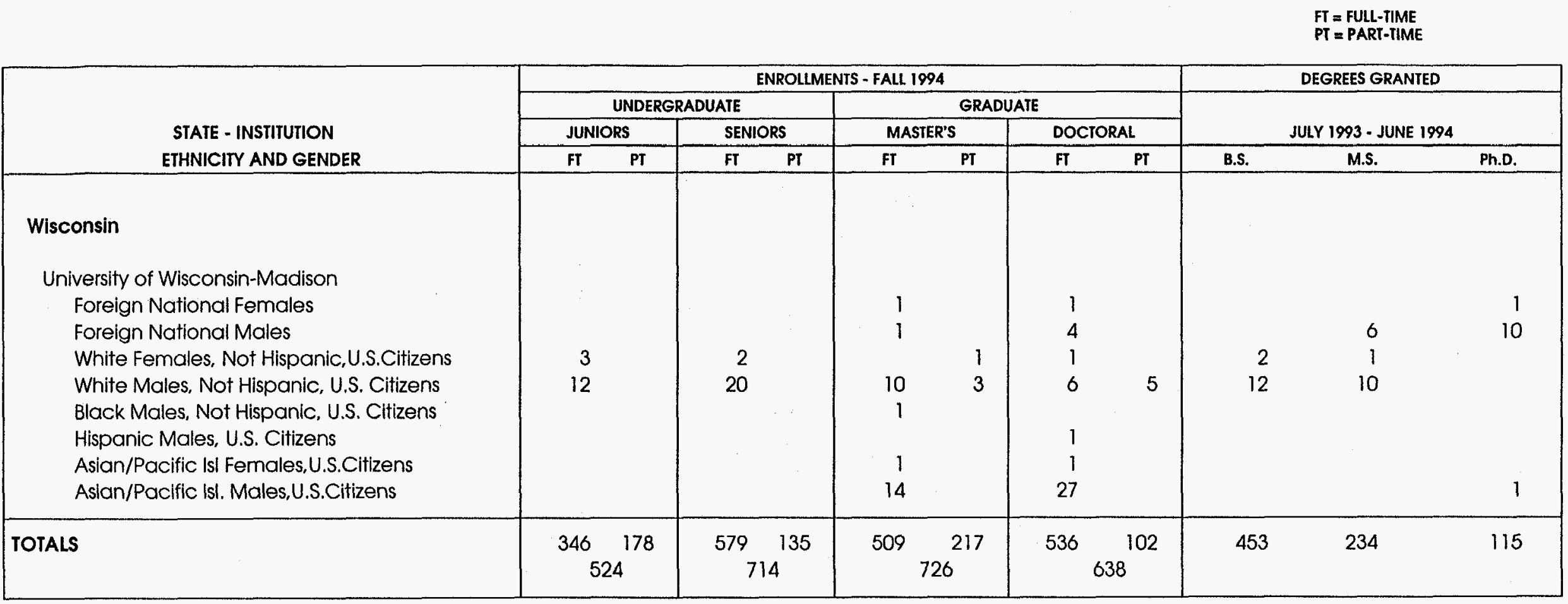

Source: U.S. Department of Energy 
APPENDIX A-8

TOTAL NUCLEAR ENGINEERING ENROUMENTS AND DEGREES

BY ETHNICITY AND GENDER, 1994

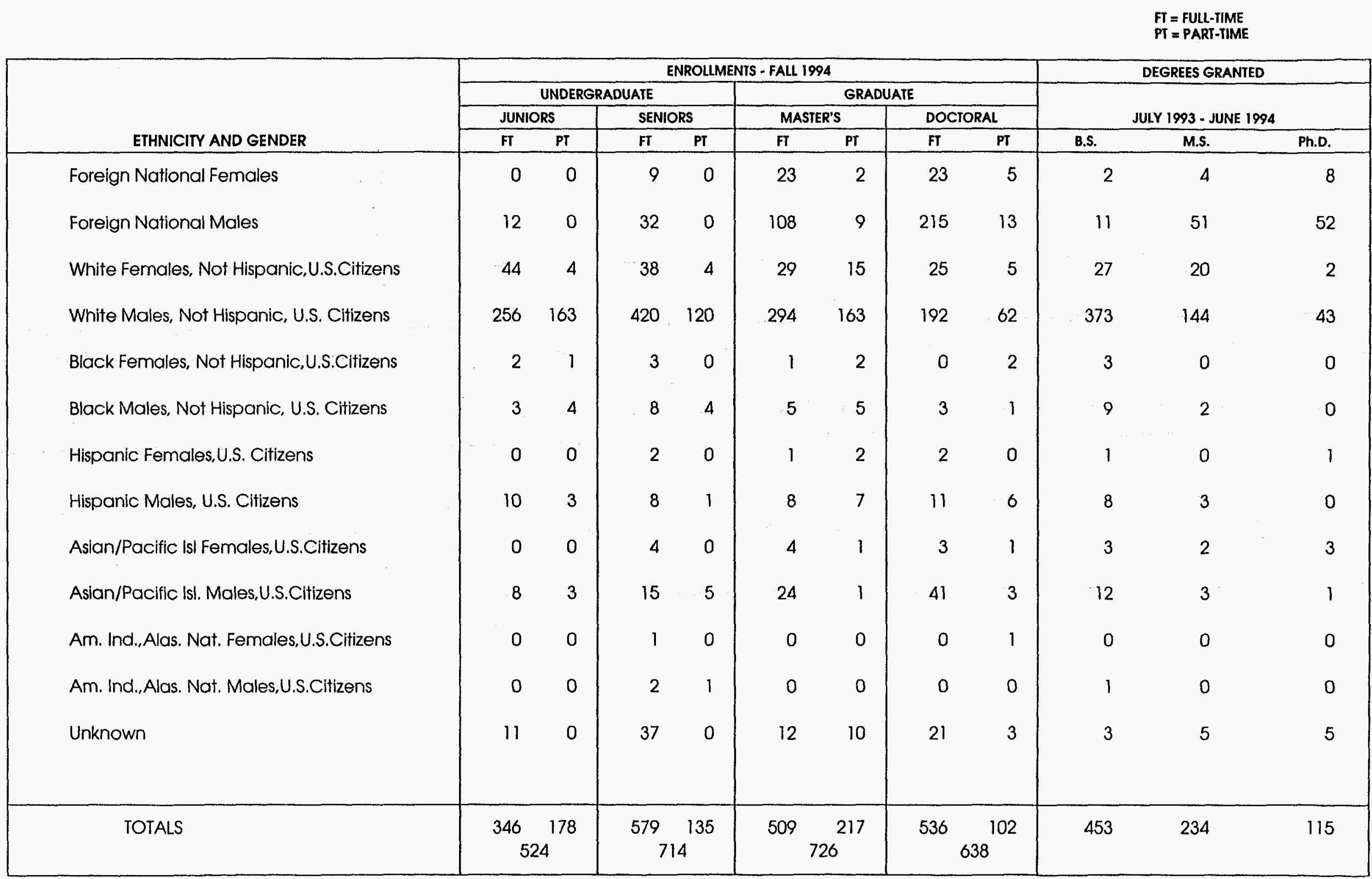


APPENDIX A-9

EMPLOYMENT OR POSTGRADUATION PLANS OF NUCLEAR ENGINEERING

GRADUATES BY DEGREE LEVEL, 1994

\begin{tabular}{|c|c|c|c|c|c|c|c|c|c|}
\hline \multirow[b]{2}{*}{$\begin{array}{l}\text { EMPLOYMENT OR } \\
\text { POSTGRADUATION PLANS }\end{array}$} & \multicolumn{3}{|c|}{ BACHELOR'S } & \multicolumn{3}{|c|}{ MASTER'S } & \multicolumn{3}{|c|}{ DOCTORAL } \\
\hline & NUMBER & PERCENT & $\begin{array}{c}\text { NUMBER OF } \\
\text { FOREIGN } \\
\text { NAIIONALS }\end{array}$ & NUMBER & PERCENT & $\begin{array}{l}\text { NUMBER OF } \\
\text { FOREIGN } \\
\text { NATIONALS }\end{array}$ & NUMBER & PERCENT & $\begin{array}{c}\text { NUMBER OF } \\
\text { FOREIGN } \\
\text { NATIONALS }\end{array}$ \\
\hline Continued study & 140 & 31 & 8 & 85 & 36 & 38 & 22 & 19 & 15 \\
\hline Academic employment, U.S. & 2 & 0 & 0 & 5 & 2 & 2 & 11 & 10 & 3 \\
\hline DOE contractor facilities (M\&Os) & 13 & 3 & 0 & 20 & 9 & 1 & 12 & 10 & 1 \\
\hline State and local government employment & 1 & 0 & 0 & 0 & 0 & 0 & 5 & 4 & 4 \\
\hline Nuclear utility employment,U.S. & 87 & 19 & 0 & 24 & 10 & 0 & 1 & 1 & 1 \\
\hline Employment with foreign employer & 0 & 0 & 0 & 8 & 3 & 8 & 19 & 17 & 18 \\
\hline U.S. military service & 53 & 12 & 0 & 13 & 6 & 0 & 3 & 3 & 0 \\
\hline Other & 7 & 2 & 1 & 4 & 2 & 1 & 8 & 7 & 3 \\
\hline Still seeking employment & 27 & 6 & $\mathbf{I}$ & 3 & 1 & 1 & 6 & 5 & 3 \\
\hline Unknown & 78 & 17 & 3 & 14 & 6 & 0 & 16 & 14 & 6 \\
\hline TOTALS & 453 & 100 & 13 & 234 & 100 & 55 & 115 & 100 & 60 \\
\hline
\end{tabular}

NOTE: Percentages are rounded to nearest whole number.

Source: U.S. Department of Energy 
BY SURVRING ENROLMMENTS AND DEGREES

BY SURVEY REGION AND STATE, 1994

FT = FULL-TIME

\begin{tabular}{|c|c|c|c|c|c|c|c|c|c|c|c|}
\hline \multirow[b]{4}{*}{ REGION - STATE } & \multicolumn{8}{|c|}{ ENROLLMENTS - FALL 1994} & \multicolumn{3}{|c|}{ DEGREES GRANIED } \\
\hline & \multicolumn{4}{|c|}{ UNDERGRADUAIE } & \multicolumn{4}{|c|}{ GRADUATE } & & & \\
\hline & \multicolumn{2}{|c|}{ JUNIORS } & \multicolumn{2}{|c|}{ SENIORS } & \multicolumn{2}{|c|}{ MASTER'S } & \multicolumn{2}{|c|}{ DOCTORAL } & \multicolumn{3}{|c|}{ JULY 1993 - JUNE.1994 } \\
\hline & $\mathbf{F}$ & PT & FT & PT & FT & PI & $\mathbf{F T}$ & PT & B.S. & M.S. & Ph.D. \\
\hline \multicolumn{12}{|l|}{ REGION I - NEW ENGLAND } \\
\hline Maine & 0 & 0 & 5 & 0 & 0 & 0 & 0 & 0 & 3 & 0 & 0 \\
\hline Massachusetts & 26 & 0 & 24 & 0 & 65 & 9 & 62 & 0 & 16 & 26 & 20 \\
\hline TOTAL & 26 & 0 & 29 & 0 & 65 & 9 & 62 & 0 & 19 & 26 & 20 \\
\hline \multicolumn{12}{|l|}{ REGION II - MIDDLE ATLANTIC } \\
\hline Maryland & 29 & 174 & 25 & 122 & 6 & 10 & 10 & 13 & 80 & 2 & 2 \\
\hline New Jersey & 1 & 0 & 1 & 0 & 1 & 1 & 0 & 0 & 1 & 1 & 0 \\
\hline New York & 54 & 0 & 74 & 0 & 20 & 44 & 26 & 7 & 59 & 22 & 9 \\
\hline Pennsylvania & 21 & 1 & 33 & 4 & 24 & 29 & 27 & 7 & 12 & 13 & 3 \\
\hline TOTAL & .105 & 175 & 133 & 126 & 51 & 84 & 63 & 27 & 152 & 38 & 14 \\
\hline \multicolumn{12}{|c|}{ REGION III - EAST NORTH-CENTRAL } \\
\hline llinois & 8 & 0 & 22 & 0 & 32 & 1 & 24 & 0 & 19 & 15 & 5 \\
\hline Indiana & 22 & 0 & 19 & 0 & 18 & 1 & 14 & 4 & 12 & 4 & 3 \\
\hline Michigan & 11 & 0 & 25 & 4 & 30 & 1 & 49 & 2 & 12 & 15 & 10 \\
\hline Ohio & 20 & 0 & 24 & 0 & 45 & 32 & 19 & 5 & 42 & 12 & 7 \\
\hline Wisconsin & 15 & 0 & 22 & 0 & 28 & 4 & 41 & 5 & 14 & 17 & 12 \\
\hline TOTAL & 76 & 0 & 112 & 4 & 153 & 39 & 147 & 16 & 99 & 63 & 37 \\
\hline \multicolumn{12}{|c|}{ REGION IV - WEST NORTH-CENTRAL } \\
\hline lowa & 0 & 0 & 0 & 0 & 5 & 1 & 7 & 0 & 3 & 2 & 0 \\
\hline Kansas & 3 & 0 & 25 & 0 & 8 & 1 & 5 & 1 & 15 & 4 & 0 \\
\hline Missouri & 14 & 0 & 21 & 0 & 13 & 5 & 18 & 0 & 8 & 8 & 0 \\
\hline TOTAL & 17 & 0 & 46 & 0 & 26 & 7 & 30 & 1 & 26 & 14 & 0 \\
\hline \multicolumn{12}{|l|}{ REGION V - SOUTH ATLANTIC } \\
\hline Florida & 13 & 0 & 24 & 0 & 32 & 14 & 20 & 9 & 27 & 7 & 8 \\
\hline
\end{tabular}


APPENDIX A-10

BY SURVEY REGION AND STATE, 1994

$F T=$ FULL-TIME

T = PART-TIME

\begin{tabular}{|c|c|c|c|c|c|c|c|c|c|c|c|}
\hline \multirow[b]{4}{*}{ REGION - STATE } & \multicolumn{8}{|c|}{ ENROLLMENIS - FALL 1994} & \multicolumn{3}{|c|}{ DEGREES GRANTED } \\
\hline & \multicolumn{4}{|c|}{ UNDERGRADUAIE } & \multicolumn{4}{|c|}{ GRADUATE } & & & \\
\hline & \multicolumn{2}{|c|}{ JUNIORS } & \multicolumn{2}{|c|}{ SENIORS } & \multicolumn{2}{|c|}{ MASTER'S } & \multicolumn{2}{|c|}{ DOCTORAL } & \multicolumn{3}{|c|}{ JULY 1993 - JUNE.1994 } \\
\hline & FT & PT & $\mathbf{F T}$ & PT & $\mathbf{F T}$ & PT & FT & PT & B.S. & M.S. & Ph.D. \\
\hline \multicolumn{12}{|l|}{ REGION V - SOUTH ATLANTIC } \\
\hline Georgia & 9 & 0 & 15 & 1 & 17 & 5 & 22 & 7 & 12 & 4 & 9 \\
\hline North Carolina & 15 & 0 & 31 & 0 & 22 & 0 & 29 & 0 & 11 & 14 & 1 \\
\hline Virginia & 1 & 0 & 10 & 0 & 11 & 7 & 7 & 2 & 5 & 1 & 1 \\
\hline TOTAL & 38 & 0 & 80 & 1 & 82 & 26 & 78 & 18 & 55 & 26 & 19 \\
\hline \multicolumn{12}{|c|}{ REGION VI - EAST SOUTH-CENTRAL } \\
\hline Kentucky & 0 & 0 & 3 & 0 & 0 & 0 & 0 & 0 & 4 & 0 & 0 \\
\hline Mississippi & 0 & 0 & 6 & 1 & 0 & 0 & 0 & 0 & 7 & 2 & 0 \\
\hline Tennessee & 20 & 0 & 26 & 0 & 20 & 2 & 16 & 1 & 13 & 11 & 0 \\
\hline TOTAL & 20 & 0 & 35 & 1 & 20 & 2 & 16 & 1 & 24 & 13 & 0 \\
\hline \multicolumn{12}{|c|}{ REGION VII - WEST SOUTH-CENTRAL. } \\
\hline Arkansas & 0 & 0 & 2 & 0 & 0 & 3 & 1 & 0 & 3 & 1 & 0 \\
\hline Louisiana & 0 & 0 & 0 & 0 & 4 & 0 & 3 & 0 & 0 & 2 & 0 \\
\hline Texas & 21 & 0 & 31 & 0 & 33 & 12 & 29 & 3 & 10 & 11 & 3 \\
\hline TOTAL & 21 & 0 & 33 & 0 & 37 & 15 & 33 & 3 & 13 & 14 & 3 \\
\hline \multicolumn{12}{|l|}{ REGION VIII - MOUNIAIN } \\
\hline Arizona & 8 & 2 & 21 & 1 & 17 & 7 & 12 & 5 & 13 & 8 & 5 \\
\hline Idaho & 3 & 1 & 3 & 0 & 9 & 13 & 2 & 11 & 6 & 2 & 0 \\
\hline New Mexico & 5 & 0 & 9 & 2 & 13 & 14 & 15 & 17 & 5 & 7 & 3 \\
\hline Utah & 2 & 0 & 14 & 0 & 6 & 0 & 7 & 0 & 9 & 4 & 3 \\
\hline TOTAL & 18 & 3 & 47 & 3 & 45 & 34 & 36 & 33 & 33 & 21 & 11 \\
\hline \multicolumn{12}{|l|}{ REGION IX - PACIFIC } \\
\hline California & 20 & 0 & 53 & 0 & 25 & 0 & 61 & 0 & 23 & 9 & 9 \\
\hline Oregon & 5 & 0 & 11 & 0 & 5 & 0 & 6 & 2 & 9 & 9 & 1 \\
\hline
\end{tabular}


NUCLEAR ENG APPENDIX A-10

BY SURVEY REGION AND STATE, 1994

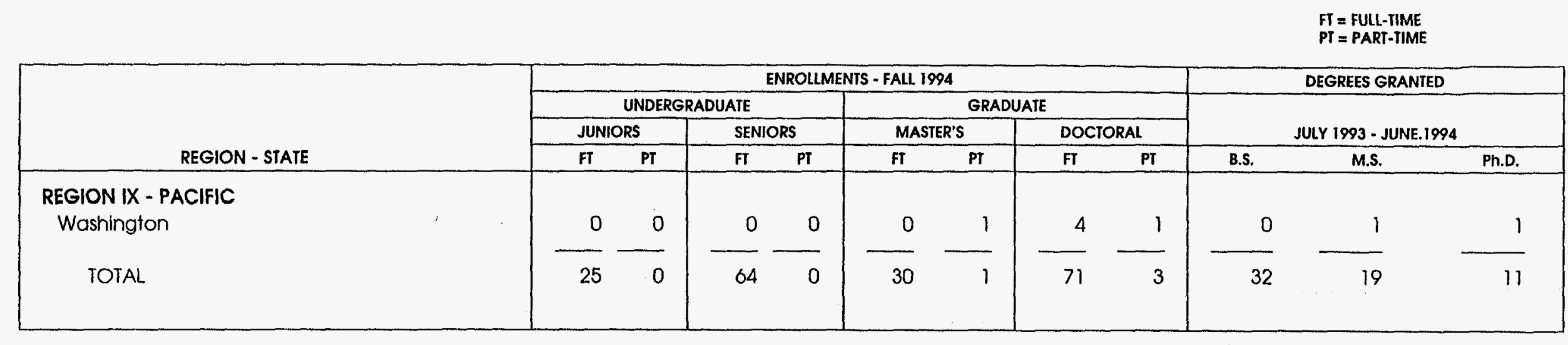

Source: U.S. Department of Energy 
APPENDIX A-11

NUCLEAR ENGINEERING AS A PERCENTAGE OF ALL ENGINEERING DEGREES

BY DEGREE LEVEL, 1974-1994

\begin{tabular}{|c|c|c|c|c|c|c|c|c|c|}
\hline \multirow[b]{2}{*}{$\begin{array}{c}\text { YEAR } \\
\text { ENDING } \\
\text { JUNE } 30 \\
\end{array}$} & \multicolumn{3}{|c|}{ BACHELOR'S } & \multicolumn{3}{|c|}{ MASTER'S } & \multicolumn{3}{|c|}{ DOCTORAL } \\
\hline & $\begin{array}{l}\text { ALL } \\
\text { ENGINEERING }\end{array}$ & $\begin{array}{l}\text { NUCLEAR } \\
\text { ENGINEERING }\end{array}$ & $\begin{array}{l}\text { PERCENT } \\
\text { NUCLEAR } \\
\text { ALL ENG. }\end{array}$ & $\begin{array}{l}\text { ALL } \\
\text { ENGINEERING }\end{array}$ & $\begin{array}{c}\text { NUCLEAR } \\
\text { ENGINEERING }\end{array}$ & $\begin{array}{c}\text { PERCENT } \\
\text { NUCLEAR } \\
\text { ALLENG. }\end{array}$ & $\begin{array}{l}\text { ALL } \\
\text { ENGINEERING }\end{array}$ & $\begin{array}{l}\text { NUCLEAR } \\
\text { ENGINEERING }\end{array}$ & $\begin{array}{c}\text { PERCENT } \\
\text { NUCLEAR } \\
\text { ALL ENG. }\end{array}$ \\
\hline 1974 & 41407 & 661 & 1.60 & 15885 & 465 & 2.93 & 3362 & 128 & 3.81 \\
\hline 1975 & 38210 & 599 & 1.57 & 15549 & 468 & 3.01 & 3138 & 103 & 3.28 \\
\hline 1976 & 37970 & 734 & 1.93 & 16506 & 468 & 2.84 & 2977 & 146 & 4.90 \\
\hline 1977 & 40094 & 805 & 2.01 & 16517 & 554 & 3.35 & 2814 & 126 & 4.48 \\
\hline 1978 & 46091 & 863 & 1.87 & 16182 & 486 & 3.00 & 2573 & 112 & 4.35 \\
\hline 1979 & 52598 & 828 & 1.57 & 16032 & 463 & 2.89 & 2815 & 117 & 4.16 \\
\hline 1980 & 58742 & 732 & 1.25 & 17243 & 366 & 2.12 & 2751 & 116 & 4.22 \\
\hline 1981 & 62935 & 692 & 1.10 & 17914 & 315 & 1.76 & 2841 & 132 & 4.65 \\
\hline 1982 & 66990 & 681 & 1.02 & 18289 & 342 & 1.87 & 2887 & 127 & 4.40 \\
\hline 1983 & 72240 & 654 & 0.91 & 19600 & 325 & 1.66 & 3023 & 124 & 4.10 \\
\hline 1984 & 76931 & 660 & 0.86 & 21226 & 316 & 1.49 & 3234 & 132 & 4.08 \\
\hline 1985 & 77964 & 612 & 0.78 & 22484 & 306 & 1.36 & 3384 & 113 & 3.34 \\
\hline 1986 & 78178 & 572 & 0.73 & 23025 & 283 & 1.23 & 3686 & 120 & 3.26 \\
\hline 1987 & 75735 & 508 & 0.67 & 24290 & 295 & 1.21 & 4175 & 97 & 2.32 \\
\hline 1988 & 71386 & 485 & 0.68 & 25616 & 232 & 0.91 & 4571 & 114 & 2.49 \\
\hline 1989 & 68824 & 454 & 0.66 & 26412 & 287 & 1.09 & 5017 & 102 & 2.03 \\
\hline 1990 & 65967 & 436 & 0.66 & 27034 & 220 & 0.81 & 5424 & 133 & 2.45 \\
\hline 1991 & 63986 & 412 & 0.64 & 27754 & 222 & 0.80 & 5680 & 122 & 2.15 \\
\hline 1992 & 63653 & 384 & 0.60 & 28540 & 212 & 0.74 & 5958 & 135 & 2.27 \\
\hline 1993 & 65001 & 411 & 0.63 & 31104 & 256 & 0.82 & 6198 & 121 & 1.95 \\
\hline 1994 & 64946 & 453 & 0.70 & 31943 & 234 & 0.73 & 6458 & 115 & 1.78 \\
\hline
\end{tabular}

Source: Data for all engineering supplied by the Engineering Workforce Commission, (Formerly, the Engineering Manpower Commission)

Washington, D.C. Nuclear engineering degrees are from the Survey of "Nuclear Engineering Enrollments and Degrees. 1994". 


\section{Nuclear Engineering}

AZ - University of Arizona - UG $G$

CA - University of California, Los Angeles - $G$

FL - University of Florida - UG $G$

ID - Idaho State University - UG G

IL - University of Illinois - UG $G$

IA - lowa State University - UG $G$

LA - Louisiana State University - $G$

MA - Massachusetts Institute of Technology - UG $G$

MI - University of Michigan - US $G$

MO - University of Missouri, Rolla - UG $G$

NM - University of New Mexico - UG $G$

NY - Cornell University - $G$

NY - United States Military Academy - UG

$\mathrm{OH}$ - Air Force Institute of Technology - $G$

$O H$ - University of Cincinnati - UG $G$

PA - The Pennsyivania State University - UG $G$

TX - Texas A\&M University - UG G

UT - University of Utah - $G$

WA - University of Washington - G
CA - University of California, Berkeley - UG G

CA - University of California, Santa Barbara - UG $G$

GA - Georgia Institute of Technology - UG G

ID - University of Idaho/INEL - $G$

IN - Purdue University - UG $G$

KS - Kansas State University - UG $G$

MD - University of Maryland - UG $G$

MA - University of Massachusetts Lowell - UG $G$

MS - Mississippi State University - UG G

MO - University of Missouri-Columbia - $G$

NY - Columbia University - $G$

NY - Rensselaer Polytechnic Institute - UG $G$

NC - North Carolina State University - UG $G$

$\mathrm{OH}$ - The Ohio State University - G

$O R$ - Oregon State University - UG G

TN - University of Tennessee - UG $G$

TX-University of Texas at Austin - $G$

$V A$ - University of Virginia - UG $G$

W) - University of Wisconsin-Madison - UG $G$

\section{Chemical, with Nuclear Option}

CA - University of California, Santa Barbara - $G$ MA - Worcester Polytechnic Institute - UG

IN - University of Notre Dame - UG

NY - United States Military Academy - UG

NY - Clarkson University - UG

UT - University of Utah - UG $G$

$\mathrm{OH}$ - Youngstown State University - UG

\section{Engineering Science, with Nuclear Option}




\title{
APPENDIXA-12 \\ INSTITUTIONS WITH UNDERGRADUATE AND GRADUATE PROGRAMS
}

OR OPTIONS IN NUCLEAR ENGINEERING, FALL 1994

\section{Electrical, with Nuclear Opfion}

CA - University of California, Berkeley - UG

$\mathrm{OH}$ - Youngstown State University - UG

IN - University of Notre Dame - UG

UT - University of Utah - UG

IN - Tennessee Technological University - UG $G$

\section{Mechanical, with Nuclear Option}

AR - University of Arkansas - UG $G$

CA - University of California, Los Angeles - UG

IN - University of Notre Dame - UG

MA - Worcester Polytechnic Institute - UG

NY - Manhattan College - UG $G$

UT - University of Utah - UG $G$

CA - University of California, Berkeley - UG

CA - University of California, Santa Barbara - $G$

KY - University of Kentucky - UG

NY - Clarkson University - UG

$\mathrm{OH}$ - The Ohio State University - UG

\section{Civil, with Nuclear Option}

NY - Clarkson University - UG

\section{Engineering or Applied Physics, with Nuclear Opt}

NJ - Stevens Institute of Technology - UG G

\section{General Engineering, with Nuclear Option}

ID - Idaho State University - UG

\author{
Marine, with Nuclear Option
}

MD - U.S. Naval Academy - UG

\section{Materials, with Nuclear Option}

CA - University of California, Santa Barbara - $G$ 
Math/Operations Research, with Nuclear Option

NY - United States Military Academy - UG

\section{Nuclear Sclence}

MD - Univ. of Maryland University College - UG

Physics, with Nuclear Option

ME - University of Maine - UG

NY - United States Military Academy - UG

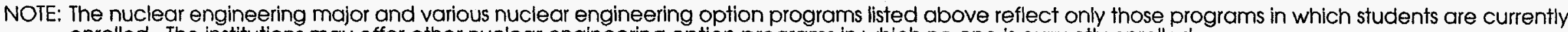
enrolled. The institutions may offer other nuclear engineering option programs in which no one is currently enrolled.

Source: U.S. Department of Energy. 
APPENDIX B. ADDRESSES 
APPENDIX B-1

ADDRESSES OF INSTITUTIONS IN SURVEY UNIVERSE, 1994

University of Arkansas

Dept. of Mechanical Engineering

Fayetteville, AR 72701

University of California, Berkele

Dept. of Nuclear Engineering

Berkeley, CA 94720

\section{University of Florida}

Nuclear Engineering Sciences

Gainesville FL 32611

Idaho State University

College of Engineering

pocatello, ID 83209-0009

Purdue University

School of Nuclear Engineering

West Lafayette, IN 47907

University of Kentucky

Dept. of Mechanical Engineering

Lexington, $\mathrm{KY}$ 40506-0046

University of Massachusefts Lowell

Nuclear Engineering Prograrn
Arizona State University

Nuclear Engineering Science

College of Engineering \& Applied Science

Tempe, A7 85287-5706

University of California, Los Angeles

Dept.of Mechanical, Aerospace and

Nuclear Engineering

Los Angeles, CA $90024-1597$

Georgia Institute of Technology

Program in Nuclear Engineering

Mechanical Engineering Department

University of Idaho/INEL

Joint Nuclear Engineering Program

idaho Falls, ID 83402

University of Notre Dame

Department of Aerospace and

Mechanical Engineering

Notre Dame, IN 46556

Louisiana State University

cience Center

Baton Rouge, LA 70803-5820

Worcester Polytechnic Instifute

Worcester, MA 01609
University of Arizono

Nuclear \& Energy Engineering

Attn: Dorothy Graves

Tucson, AZ 85721

University of California, Santa Barbara Dept. of Chemical \& Nuclear Engineerin Santa Barbara. CA 93106

lowa State University Nuclear Engineering Program Ames, IA 50011-2241

University of Illinois Dept. of Nuclear Engineering Urbana, IL 6180

Kansas State University Dept. of Nuclear Engineering Manhattan, KS 66506-2503

Massachusetts Institute of Technology Deptartment of Nuclear Engineering Cambridge, MA 0213

U.S. Naval Academy Naval Systems Engineering (NAOME) Annapolis, MD 21402-5000 
Univ. of Maryland University College Office of Special Program

College Park, MD 20742

University of Michigan

Department of Nuclear Engineering

Ann Arbor, MI 48109-2100

Mississippi State University

Dept. of Mechanical Engineering

Mississippi State., MS 39762

University of New Mexico

Dept. of Chemical \& Nuclear Engineering

Albuquerque. NM 87131

\section{Cornell University}

Nuclear Science \& Engineering Program

United States Military Acaderny

Department of Physics
West Point, NY 10996-1790

University of Cincinnati

Dept. of Mechanical, Industrial

and Nuclear Engineering
University of Maryland

Nuclear Engineering Program

Dept. of Materials \& Nuclear Engineering

College Park, MD 20742

University of Missouri, Rolla

Department of Nuclear Engineering

Rolla, MO 65401

North Carolina State University

Department of Nuclear Engineering

Raleigh, NC 27695-7909

Clarkson University

Dept. of Chemical Engineering

Potsdam. NY 13699-5705

Manhattan College

Department of Mechanical Engineering

Air Force Institute of Technology

Nuclear Engineering Program

Department of Engineering Physics

Dayton. $\mathrm{OH}$ 45433-7765

Youngstown State University

Dept. of Chemical and Nuclear Engineering

School of Engineering
University of Maine

Department of Physics

Orono, ME 04469

University of Missouri-Columbia Department of Nuclear Engineering

Stevens Institute of Technology Dept. of Engineering Physics

Hoboken, NJ 07030

Columbla University
Dept. of Applied Physics
New York, NY 10027

Rensselaer Polytechnic Institute Department of Nuclear Engineering Troy, NY 12180-3590

The Ohio State University Nuclear Engineering Program
Columbus, $\mathrm{OH} 43210-1107$

Oregon State University Department of Nuclear Engineering Corvallis, OR $97331-5902$ 

APPENDIX C. SURVEY MATERIALS 


\section{Department of Energy \\ Washington, DC 20585}

October 14, 1994

Dear Professor:

Enclosed is the annual Nuclear Engineering Enrollments and Degrees Survey sponsored by the Department of Energy. The survey is designed to include those programs offering a major in nuclear engineering or course work equivalent to a major in other engineering disciplines that prepare the graduates to perform as nuclear engineers. This survey provides data on nuclear engineering enrollments and degrees for use in labor market analyses, information on education programs for students, and information on new graduates to employers, government agencies, academia and professional societies. We appreciate your voluntary effort in helping us provide this information.

Currently, the labor market for nuclear engineers is undergoing major shifts. Nuclear electric utilities employment has stablized, nuclear defense and security employment has decreased, environmental restoration and waste management employment has expanded, and demand in the general economy continues to be relatively weak for science and engineering fields. Overall, job openings for new graduates in nuclear engineering result largely from job openings for replacement needs from attrition. At the same time, enrollments in nuclear engineering continue to decline. If the enrollment decline persists over the next several years, the number of new graduates may not be sufficient even to meet the job openings for replacement needs. Thus, it continues to be important to collect nuclear engineering education data in order to assess likely future labor market trends.

Instructions for completing the form are on the back of this letter, and a selfaddressed stamped envelope is provided for your convenience. We would appreciate your response within four weeks. If you have questions, please contact Duveen L. Shirley, Science/Engineering Education Division, Oak Ridge Institute for Science and Education (615) 576-3409, Fax (615) 241-2727, Internet SHIRLEYD@ORAU.GOV.

MLW:ds

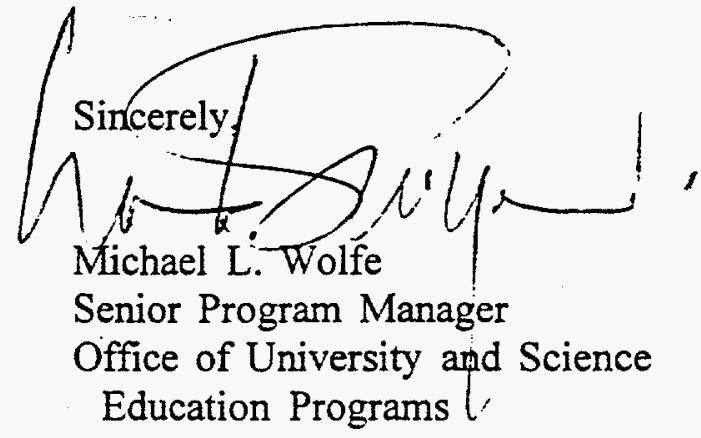

Enclosures 
Note: Information provided through this survey is not considered to be confidential and may be disclosed to the public.

\section{INSTRUCTIONS AND DEFINITIONS FOR COMPLETING DOE FORM ER-616}

WHO SHOULD COMPLETE AND SUBMIT FORM: All departments with programs offering a major in nuclear engineering or in other engineering disciplines (e.g., mechanical or chemical) that provide nuclear engineering course work equivalent to a major in nuclear engineering (nuclear engineering option) that would prepare graduates TO FUNCTION AS NUCLEAR ENGINEERS. Exception: any health physics or radiation protection option within an engineering curriculum should be reported on DOE Form ER-617, which is available from the address or telephone number given below. Each major program should be reported on the appropriate line or on separate lines in the write-in space. Very small specialties should be combined.

WHEN AND WHERE TO SUBMIT FORM: The form should be submitted within four weeks of receipt or earlier, depending upon your institution's enrollment schedule. Send completed form to:

Duveen L. Shirley

Oak Ridge Institute for Science and Education

P.O. Box 117

Oak Ridge, Tennessee 37831-0117

(615) 576-3409, Fax (615) 241-2727,

Internet SHIRLEYD@ORAU.GOV

A Self-addressed, postage-paid envelope is enclosed for your convenience.

SPECIAL INSTRUCTIONS: Report only students enrolled in a degree program. Count on-campus semester co-op students (see definition below) as full-time students. If exact figures are not available, give your best estimate and indicate by an "E" after each number that is estimated. (Report numbers in spaces where appropriate leaving all other spaces blank.)

\section{DEFINITIONS}

Junior: A student who is more than one and less than two academic years short of a Bachelor's degree or equivalent.

Senior: A student who is one year or less short of a Bachelor's degree or equivalent.

Master's Candidate: A student enrolled in a program leading to any degree above the B.S. or B.A. level and below the Ph.D. level.

CO-OP Student: A student who alternates sessions of schooling with sessions of employment in a position related to the academic specialty.

Foreign National: Any student who is a citizen of another country and who has not applied for U.S. citizenship.

White not of Hispanic origin: A person having origin in any of the original peoples of Europe, North Africa, or the Middle East.

Black not of Hispanic origin: A person having origin in any of the black racial groups of Africa.

Hispanic: A person of Mexican, Puerto Rican, Cuban, Central or South American, or other Spanish culture or origin regardless of race.

Asian or Pacific Islander: A person having origin in any of the original peoples of the Far East, Southeast Asia, the Indian subcontinent, or the Pacific Islands.

American Indian or Alaskan Native: A person having origin in any of the original peoples of North America and who maintains cultural identification through tribal affiliation or community recognition. 


\section{Affix label if correct; otherwise, please type correct address here.}

7

Contact Person

Department(s)

Signature

While you are not required to respond, your cooperation is needed to make the results comprehensive,

accurate, and timely. Information will NOT be held confidential, and is supplied to government and

L

(Please report only those students in nuclear engineering programs or other engineering disciplines with nuclear courses sufficiont to prepare them to perform as nuclear engineers.)

SECTION A - Curriculum or Option

Nuclear engineering program

Nuclear engineering option within chemical engineering program

Nuclear engineering option within engineering science program

Nuclear engineering option within electrical engineering program

Nuclear engineering option within mechanical engineering program

Other: (Specify, e.g., Industrial, Civil)

TOTALS

SECTION B - Racial, Ethnic and Gender Breakdown

Foreign nationals (Temporary Visas)

White U.S. citizens or permanent residents,

not of Hispanic origin

Black U.S. citizens or permanent residents,

not of Hispanic origin

Hispanic U.S. citizens or permanent residents

Asian or Pacific Islander U.S. citizens or

permanent residents

American Indian or Alaskan native U.S. citizens or

permanent residents

\begin{tabular}{|c|c|}
\hline & CODE \\
\hline Male & $1 \mathrm{M}$ \\
\hline Female & $1 F$ \\
\hline Male & $2 M$ \\
\hline Female & $2 F$ \\
\hline Male & $3 M$ \\
\hline Female & $3 F$ \\
\hline Male & $4 M$ \\
\hline Female & $4 F$ \\
\hline Male & $5 \mathrm{M}$ \\
\hline Female & $5 \mathrm{~F}$ \\
\hline Male & $6 M$ \\
\hline Female & $6 F$ \\
\hline
\end{tabular}

TOTALS

Fomale 1 of
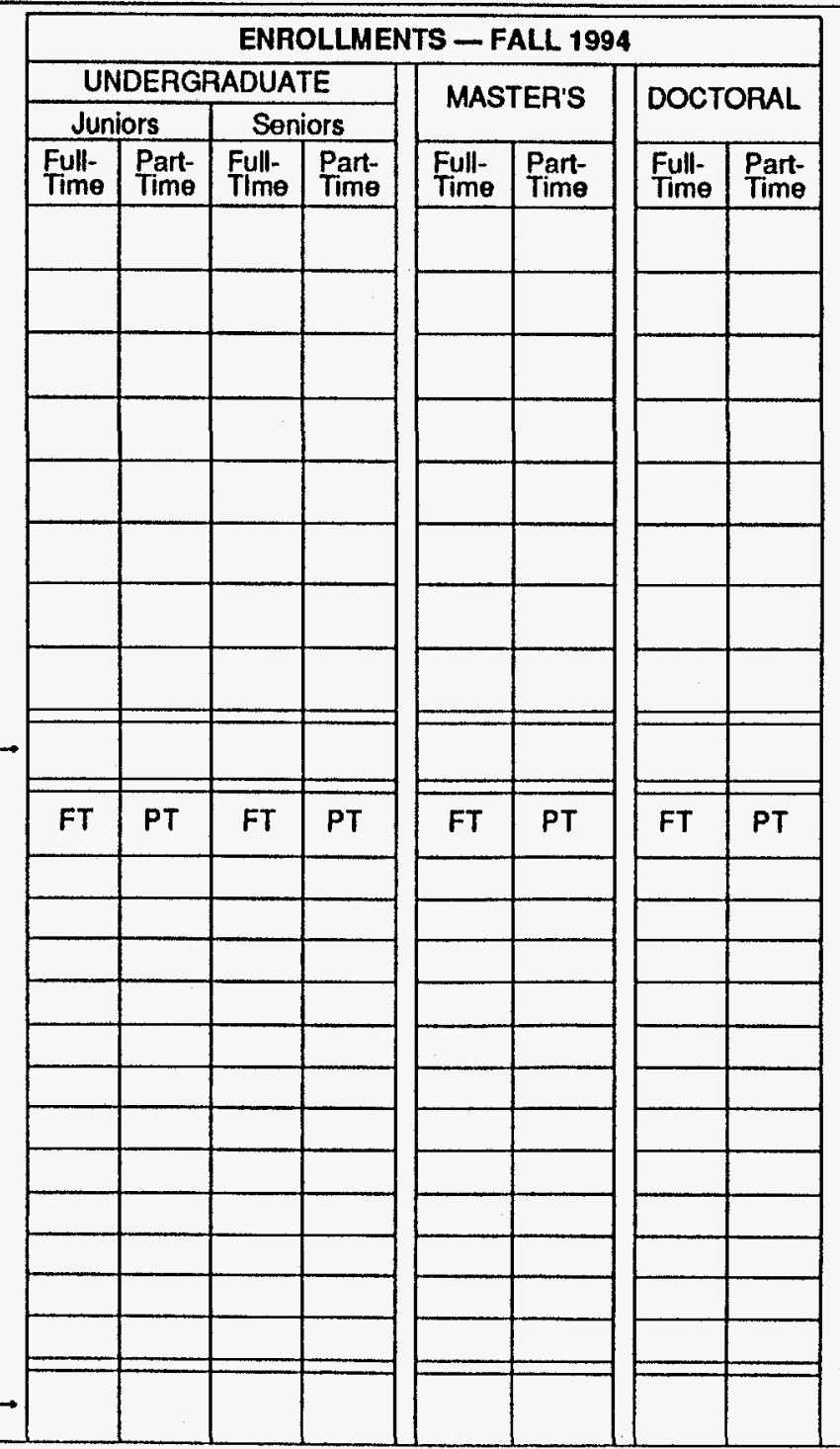

DEGREES GRANTED

July 1993 - June 1994

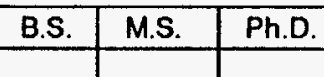

"Health physics/radiation protection enginearing students should be entered on form DOE F ER-617. (See survey instructions.) 
Institution

\begin{tabular}{|c|c|c|c|c|c|c|c|}
\hline \multirow{2}{*}{$\begin{array}{c}\text { SECTON C-Employment or Postgraduation Plans } \\
\text { For degrees granted } \\
\text { July } 1,1893 \text { through June } 30,1994\end{array}$} & \multirow[b]{2}{*}{ CODE } & \multicolumn{2}{|c|}{ BACHELOR'S } & \multicolumn{2}{|c|}{ MASTER'S } & \multicolumn{2}{|c|}{ DOCTORAL } \\
\hline & & $\begin{array}{l}\text { Total } \\
\text { Graduates }\end{array}$ & $\begin{array}{l}\text { Number of } \\
\text { Foreign } \\
\text { Nationals } \\
\text { in Totals }\end{array}$ & $\begin{array}{l}\text { Total } \\
\text { Graduates }\end{array}$ & $\begin{array}{l}\text { Number of } \\
\text { Foreign } \\
\text { Nationals } \\
\text { in Totals }\end{array}$ & $\begin{array}{c}\text { Total } \\
\text { Graduates }\end{array}$ & $\begin{array}{l}\text { Number of } \\
\text { Foreign } \\
\text { Nationals } \\
\text { in Totals }\end{array}$ \\
\hline $\begin{array}{l}\text { Continued study for higher degree or postdoctoral } \\
\text { study. (Include students employed part-time.) }\end{array}$ & 1 & & & & & & \\
\hline Academic employment, U.S. & 2 & & & & & & \\
\hline Foderal government employment, U.S. & 3 & & & & & & \\
\hline $\begin{array}{l}\text { DOE contractor R\&D and production } \\
\text { facilities (M\&Os) }\end{array}$ & 4 & & & & & & \\
\hline State and local government employment, U.S. & 5 & & & & & & \\
\hline Nuclear utility employment, U.S. & 6 & & & & & & \\
\hline Other industrial employment, U.S. & 7 & & & & & & \\
\hline Employment with foreign employer & 8 & & & & 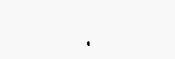 & & \\
\hline U.S. military service & 9 & & & & & & \\
\hline Other (specily) & 10 & & & & & & \\
\hline Seoking employment & 11 & & & & & & \\
\hline Unknown & 12 & & & & & & \\
\hline $\begin{array}{ll}\text { TOTALS } & \begin{array}{l}\text { ("Total Graduates" column at each degree } \\
\text { should agree with "Degrees Granted" totals } \\
\text { the front side.) }\end{array}\end{array}$ & & & & & & & \\
\hline
\end{tabular}

Remarks: 